\title{
25 Research Soure \\ Ethnomedicinal Knowledge of Pwo People in Northern Thailand
}

\section{Wittaya Pongamornkul}

Queen Sirikit Botanic Garden

Nattee Muangyen

Queen Sirikit Botanic Garden

\section{Benjaporn Phookaphin}

Queen Sirikit Botanic Garden

\section{Prateep Panyadee}

Queen Sirikit Botanic Garden

Angkhana Inta ( $\sim$ aungkanainta@hotmail.com)

Chiang Mai University

\section{Research}

Keywords: Pwo Karen, medicinal plants, local plants, traditional knowledge, ethnobotanical indices

Posted Date: April 13th, 2020

DOI: https://doi.org/10.21203/rs.3.rs-21537/v1

License: (c) (i) This work is licensed under a Creative Commons Attribution 4.0 International License. Read Full License 


\section{Abstract}

Background: Northern Thailand is the home of many ethnic groups who possess great knowledge of ethnomedicine, so it is the location with the highest number of ethnobotanical studies in Thailand. However, the Pwo have never been included in. Therefore, this study is the first ethnobotanical study of Pwo people.

Methods: In this study, 50 Pwo informants from 8 villages in Omkoi district were interviewed about their ethnomedicinal knowledge. They were asked about the vernacular name(s) and uses of plant species found in their villages and nearby forests.

Results : A total of 122 ethnomedicinal species with 930 use-reports were recorded. Leaves were the most preferred plant part and decoction was the most preferred preparation method. Herbs were the most favored life form of ethnomedicinal plants used by Pwo people. Asteraceae, Fabaceae, and Zingiberaceae were the three most dominant plant families, which contributed $22 \%$ of the total species. Most plants with high Use value (UV) values were common species which also found and reported in other ethnobotanical studies however, the exception was Garcinia pedunculata Roxb. ex Buch.-Ham. Although only a single individual was found in nearby forests, but it was commonly used by Pwo people in the studied sites. The five ailments with high recorded IAR included: injuries, muscular-skeletal system, pain, respiratory systems, and digestive systems. These ailments were directly related to the life-style of Pwo people.

Conclusion : The comparison between the species found in this study and a study of Sgaw Karen in a nearby area showed that their knowledge differed from each other in both species and their uses despite their close proximity. The results emphasize the importance and the need of ethnobotanical studies although in the areas where many studied have been carried out. The more understanding of the traditional knowledge would increase our chance of understanding and identification of medicinal properties of plants. The results would lead to the discovery and development of new pharmaceutical substances to improve human life quality.

\section{Background}

Since the dawn of civilization, humans have had to cope with various diseases. Medicinal plants were one of the keys to prolonging human life [1], and civilization. Until now, medicinal plants still have an important role in daily life, especially for people who live in rural areas which included about half the global population [2]. Moreover, 90\% of the population in developing countries still rely on traditional medicinal plants for their primary health [3]. The uses of medicinal plants differ from place to place and ethnicity to ethnicity depending on the plant diversity around them. Southeast Asia is the harbor of biodiversity and cultural diversity which is reflected in the great variety systems of traditional medicine [4]. Ethnobotany of medicinal plants examines the relationship between biological and cultural parts of the environment [5]. Ethnobotanical study of medicinal plants is an important key to bringing local medicinal plant knowledge into the purview of science and conserving them.

Northern Thailand is home to more than 20 ethnic groups [6]. These ethnic groups possess unique ethnobotanical knowledge which differs between groups. Despite the rich diversity in both ethnicity and plants, there have been only about 20 ethnobotanical publications from northern Thailand and only seven publications about Karen [4,712]. This is the highest number for any ethnic group. However, all these Karen ethnobotanical publications focus only Sgaw Karen, which was the largest group of Karen. There was no earlier Pwo ethnobotanical publication, so this is the first publication of Pwo ethnobotanical research. 
In this study we investigated the ethnomedicinal plants used by Pwo people to i) document the ethnomedicinal knowledge of Pwo people for their ethnomedicinal plants which included the details of preparations and application, ii) compare the uses of medicinal plant by Pwo people to the medicinal plants used elsewhere in Thailand iii) compare the importance of each ethnomedicinal species to the Pwo communities.

\section{Materials And Methods Study sites}

The ethnobotanical investigation was carried out in eight Pwo villages in Nakian sub-district, Omkoi district, Chiang Mai province (Fig. 1). The elevations of the studied sites ranged between 938-1460 m.a.s.l. (Table 1). All villages were located in valleys surrounded by dry evergreen forest. However, Kong Po Tai was also close to mixed deciduous forest because this village was located at low elevation. Nakian sub-district was located about $30 \mathrm{~km}$ from the center of Omkoi district. U-toom village was the center of the sub-district while other villages were located about 7-25 km away from this village. The villages were connected by a dusty road which sometimes was impassable in the rainy season. Swidden rice agriculture was practiced in all studied villages along with paddy rice farming. In two villages only swidden agriculture was practiced (Table 1). All Pwo people in this study are animists and Buddhist. 
Table 1

Basic information and number of informants in eight Pwo villages in Na Kian sub-district, Omkoi district, Chiang Mai Province, Thailand where the ethnomedicinal investigation was carried on.

\begin{tabular}{|c|c|c|c|c|c|}
\hline $\begin{array}{l}\text { Village } \\
\text { name }\end{array}$ & Coordinates & $\begin{array}{l}\text { Elevation } \\
\text { (m.a.s.) }\end{array}$ & Vegetation* & $\begin{array}{l}\text { Distance from Sub- } \\
\text { district center (k.m.) }\end{array}$ & Rice practice \\
\hline Bai $\mathrm{Na}$ & $\begin{array}{l}\text { N17 } 17^{\circ} 51^{\prime} 43.74^{\prime \prime} \\
\text { E098 } 08^{\prime} 17.88^{\prime \prime}\end{array}$ & 1180.0 & DEF & 20 & Swidden \\
\hline $\begin{array}{l}\text { Kong Po } \\
\text { Tai }\end{array}$ & $\begin{array}{l}\text { N17 } 17^{\circ} 50^{\prime} 21.54^{\prime \prime} \\
\text { E098 } 10^{\prime} 54.08^{\prime \prime}\end{array}$ & 938.0 & DEF/MDF & 10 & Paddy/Swidden \\
\hline $\begin{array}{l}\text { Mae } \\
\text { Khong }\end{array}$ & $\begin{array}{l}\mathrm{N} 17^{\circ} 43^{\prime} 18.84^{\prime \prime} \\
\text { E098 } 10^{\circ} 42.48^{\prime \prime}\end{array}$ & 1040.0 & DEF & 25 & Paddy/Swidden \\
\hline Na Kian & $\begin{array}{l}\mathrm{N} 17^{\circ} 47^{\prime} 31.26^{\prime \prime} \\
\text { E0980ㅇ'07.08" }\end{array}$ & 1260.0 & DEF & 20 & Paddy/Swidden \\
\hline $\begin{array}{l}\text { Sa-ngin } \\
\text { Klang }\end{array}$ & $\begin{array}{l}\text { N17 } 17^{\circ} 53^{\prime} 59.26^{\prime \prime} \\
\text { E098 } 14^{\circ} 06.47^{\prime \prime}\end{array}$ & 1243.0 & DEF & 15 & Swidden \\
\hline $\begin{array}{l}\text { Sa-ngin } \\
\text { Neua }\end{array}$ & 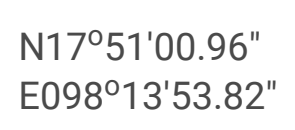 & 1400.0 & DEF & 8 & Paddy/Swidden \\
\hline Tang Tee & $\begin{array}{l}\text { N17 } 5050^{\prime} 51.48^{\prime \prime} \\
\text { E098 } 10^{\prime} 58.86^{\prime \prime}\end{array}$ & 950.0 & DEF & 7 & Paddy/Swidden \\
\hline U-toom & $\begin{array}{l}\text { N17 } 17^{\circ} 50^{\prime} 55.92^{\prime \prime} \\
\text { E098 } 12^{\prime} 55.44^{\prime \prime}\end{array}$ & 1460.0 & DEF & 0 & Paddy/Swidden \\
\hline
\end{tabular}

\section{Data collection}

Field surveys were made every two month from October 2017 to September 2019. The ethnobotanical data were collected from 4-15 informants from each village. The interviews were carried out in the Pwo language with the help of local guides. The informants were informed about the purpose of the study and asked for their consent to provide their ethnomedicinal knowledge. Field surveys were conducted in villages, home gardens, agricultural areas, and nearby forests. During field surveys, the ethnomedicinal data were recorded using a semi-structured interview. The recorded data included the vernacular names, uses of the plant, the parts used, methods of preparation, and routes of administration.

\section{Plant identifications and categorization}

Between the ethnobotanical surveys, plants were photographed and the collected for future identification. The identification was done at the Queen Sirikit Botanic Garden Herbarium (QBG herbarium) and the specimens were deposited there. The botanical names and families were verified by The Plant List version 1.1 based on APGIII [13]. 
The ailment categories and parts used followed the Economic Botany Data Collection Standard [14]. The life forms of plants were adopted from Thai Plant Names Tem Smitinand [15].

\section{Data analysis}

\section{Use value (UV)}

The UV was used to compare the importance of species used by the informants [16]. The index was calculated as $\boldsymbol{U V}=\sum \boldsymbol{U}_{\boldsymbol{i}} / \boldsymbol{n}$ where $\boldsymbol{U}_{\boldsymbol{i}}$. is the number of use-reports from each informant for a species $i$ and $n$ is the total number of informants (in this study, $n=50$ ). Use values are high when plants had more use-reports from the informant, and approach zero when there was no use-report.

\section{Informant Agreement Ratio (IAR)}

This index measured the agreement among the informants about which plants to use for any particular use category [17,18]. The index can be calculated as $\boldsymbol{I} \boldsymbol{A} R=\left(\boldsymbol{N}_{\boldsymbol{u r}}-\boldsymbol{N}_{\boldsymbol{t}}\right) /\left(\boldsymbol{N}_{\boldsymbol{u r}}-\mathbf{1}\right)_{\text {where }} \boldsymbol{N}_{\boldsymbol{u r}}$ is the number of use-reports for a particular use category and $N_{t}$ is the number of taxa used for that use category cited by all informants. The IAR value was ranged between $0-1$. The value 1 indicated that only a single species was used by all informants for a particular category while the value 0 meant each informant used different species for a use category, i.e., there was no agreement among the informants.

\section{Comparing ethnomedicinal plants used by Pwo people to medicinal plants in Thailand}

Plants used by the Pwo people were compared to the ethnomedicinal plant list for Thailand compiled by Phumthum et al. (2018) [19] from 64 reports from throughout Thailand. Plants that were identified to only genus level were excluded from this comparison.

\section{Results And Discussion}

\section{The ethnomedicinal plants used by Pwo}

A total of 122 ethnomedicinal species from 111 genera and 62 families were recorded from 50 Pwo informants (Table 2). The most dominant families were Asteraceae (10 species 8.19\%), Fabaceae (9 species 7.37\%), and Zingiberaceae (8 species 6.55\%) (Fig. 2). 
Table 2

The ethnomedicinal plants and their usages which were reported from 50 Pwo informants, from 8 villages in $\mathrm{Na}$ Kian sub-district, Omkoi district, Chiang Mai province, Thailand.

\begin{tabular}{|c|c|c|c|c|c|c|}
\hline $\begin{array}{l}\text { FAMILY } \\
\text { Scientific names }\end{array}$ & $\begin{array}{l}\text { Local } \\
\text { name }\end{array}$ & $\begin{array}{l}\text { Use } \\
\text { value } \\
\text { (UV) }\end{array}$ & $\begin{array}{l}\text { Part used and } \\
\text { application* }\end{array}$ & Preparation** & $\begin{array}{l}\text { Route of } \\
\text { administration }\end{array}$ & $\begin{array}{l}\text { Voucher } \\
\text { number }\end{array}$ \\
\hline \multicolumn{7}{|l|}{ ACANTHACEAE } \\
\hline Andrographis sp. & $\begin{array}{l}\text { Ta- } \\
\text { Phu-E- } \\
\text { Khae }\end{array}$ & 0.06 & $\begin{array}{l}\text { Lf: Wounds } \\
\text { Lf: Diabetes, } \\
\text { Cancer }\end{array}$ & $\begin{array}{l}\text { Po } \\
\text { De }\end{array}$ & $\begin{array}{l}\text { Poultices } \\
\text { Oral }\end{array}$ & $\begin{array}{l}\text { W. } \\
\text { Pongamornkul } \\
5239\end{array}$ \\
\hline Justicia adhatoda L. & $\begin{array}{l}\text { Se-Ta- } \\
\text { Ro }\end{array}$ & 0.08 & $\begin{array}{l}\text { Rt: Chest pain, } \\
\text { Stomachache } \\
\text { St, Lf: Asthma } \\
\text { Lf: Wounds }\end{array}$ & $\begin{array}{l}\text { De } \\
\text { In } \\
\text { Po }\end{array}$ & $\begin{array}{l}\text { Oral } \\
\text { Oral } \\
\text { Poultices }\end{array}$ & $\begin{array}{l}\text { W. } \\
\text { Pongamornkul } \\
03912\end{array}$ \\
\hline $\begin{array}{l}\text { Justicia gendarussa } \\
\text { Burm. f. }\end{array}$ & $\begin{array}{l}\text { Ta-Se- } \\
\text { Khae }\end{array}$ & 0.2 & $\begin{array}{l}\text { Rt: Cough } \\
\text { Wp: Muscle } \\
\text { pain } \\
\text { St, Lf: Pain } \\
\text { St, Lf: Fever }\end{array}$ & $\begin{array}{l}\mathrm{De} \\
\mathrm{Bu} \\
\mathrm{Bu} \\
\mathrm{In}\end{array}$ & $\begin{array}{l}\text { Oral } \\
\text { Poultices } \\
\text { Poultices } \\
\text { Bath }\end{array}$ & - \\
\hline $\begin{array}{l}\text { Pseuderanthemum } \\
\text { sp. }\end{array}$ & $\begin{array}{l}\text { De-Ber- } \\
\text { Du }\end{array}$ & 0.04 & $\begin{array}{l}\text { Lf: } \\
\text { Stomachache, } \\
\text { Nourishment }\end{array}$ & $\mathrm{De}$ & Oral & $\begin{array}{l}\text { W. } \\
\text { Pongamornkul } \\
4891,5695\end{array}$ \\
\hline $\begin{array}{l}\text { Thunbergia coccinea } \\
\text { Wall. ex D. Don }\end{array}$ & $\begin{array}{l}\text { Cho- } \\
\text { Ka-La } \\
\text { Mue }\end{array}$ & 0.04 & $\begin{array}{l}\text { Fl: Appetizing } \\
\text { Fl: Cough }\end{array}$ & $\begin{array}{l}\mathrm{Bu} \\
\mathrm{De}\end{array}$ & $\begin{array}{l}\text { Oral } \\
\text { Oral }\end{array}$ & $\begin{array}{l}\text { W. } \\
\text { Pongamornkul } \\
4879\end{array}$ \\
\hline $\begin{array}{l}\text { Thunbergia laurifolia } \\
\text { Lindl. }\end{array}$ & $\begin{array}{l}\text { Cho- } \\
\text { Ka-La- } \\
\text { Pho }\end{array}$ & 0.14 & $\begin{array}{l}\text { Lf: Gastritis, } \\
\text { Detoxification } \\
\text { St, Lf: } \\
\text { Hypertension } \\
\text { St: } \\
\text { Stomachache, } \\
\text { Muscle pain }\end{array}$ & $\begin{array}{l}\mathrm{De} \\
\mathrm{De} \\
\mathrm{De}\end{array}$ & $\begin{array}{l}\text { Oral } \\
\text { Oral } \\
\text { Oral }\end{array}$ & $\begin{array}{l}\text { W. } \\
\text { Pongamornkul } \\
03977\end{array}$ \\
\hline \multicolumn{7}{|l|}{ ACORACEAE } \\
\hline Acorus calamus L. & $\begin{array}{l}\text { Pho- } \\
\text { Bue- } \\
\text { Lae }\end{array}$ & 0.72 & $\begin{array}{l}\text { Rt: Flatulence, } \\
\text { Cough } \\
\text { Wp: Fever } \\
\text { Lf: } \\
\text { Stomachache }\end{array}$ & $\begin{array}{l}\mathrm{Np} \\
\mathrm{De} \\
\mathrm{Np}\end{array}$ & $\begin{array}{l}\text { Oral } \\
\text { Bath } \\
\text { Oral }\end{array}$ & $\begin{array}{l}\text { W. } \\
\text { Pongamornkul } \\
5334\end{array}$ \\
\hline
\end{tabular}

\footnotetext{
* Abbreviations for the plant parts: Bk = Bark; Bu = Bulbil; FI = Flower; Fr = Fruit; Ga = Gall; La = Latex; Lf = Leaf; Rh $=$ Rhizome; Rt = Root; Sd = Seed; St = Stem; Tu = Tuber; $\mathrm{Wp}=$ Whole plant

**Abbreviations for the preparation methods: $\mathrm{Bu}=$ Burned; $\mathrm{De}=$ Decoction; $\mathrm{In}=$ Infusion; $\mathrm{Np}=$ Non-prepared ; Po $=$ Pounded
} 


\begin{tabular}{|c|c|c|c|c|c|c|}
\hline $\begin{array}{l}\text { FAMILY } \\
\text { Scientific names }\end{array}$ & $\begin{array}{l}\text { Local } \\
\text { name }\end{array}$ & $\begin{array}{l}\text { Use } \\
\text { value } \\
\text { (UV) }\end{array}$ & $\begin{array}{l}\text { Part used and } \\
\text { application* }\end{array}$ & Preparation** & $\begin{array}{l}\text { Route of } \\
\text { administration }\end{array}$ & $\begin{array}{l}\text { Voucher } \\
\text { number }\end{array}$ \\
\hline $\begin{array}{l}\text { Sambucus javanica } \\
\text { Blume }\end{array}$ & $\begin{array}{l}\text { Choe- } \\
\text { Cha-Bi }\end{array}$ & 0.32 & $\begin{array}{l}\text { Rt: Stones, } \\
\text { Diuretic } \\
\text { Lf: Muscle } \\
\text { pain, Pain, } \\
\text { Postpartum } \\
\text { period, } \\
\text { Lf, St: } \\
\text { Hemorrhoids }\end{array}$ & $\begin{array}{l}\mathrm{De} \\
\mathrm{Bu} \\
\mathrm{De}\end{array}$ & $\begin{array}{l}\text { Oral } \\
\text { Poutices } \\
\text { Oral }\end{array}$ & $\begin{array}{l}\text { W. } \\
\text { Pongamornkul } \\
\text { et al. } 5121\end{array}$ \\
\hline \multicolumn{7}{|l|}{ AMARANTHACEAE } \\
\hline $\begin{array}{l}\text { Amaranthus blitum L. } \\
\text { subsp. oleraceus (L.) } \\
\text { Costea }\end{array}$ & $\begin{array}{l}\text { Mu- } \\
\text { Lang- } \\
\text { Du }\end{array}$ & 0.04 & $\begin{array}{l}\text { Wp: } \\
\text { Stomachache, } \\
\text { Leg pain }\end{array}$ & $\mathrm{De}$ & Oral & $\begin{array}{l}\text { W. } \\
\text { Pongamornkul } \\
4586\end{array}$ \\
\hline $\begin{array}{l}\text { Amaranthus } \\
\text { spinosus L. }\end{array}$ & $\begin{array}{l}\text { Mu- } \\
\text { Lang- } \\
\text { Du }\end{array}$ & 0.04 & $\begin{array}{l}\text { Wp: } \\
\text { Stomachache, } \\
\text { Leg pain }\end{array}$ & $\mathrm{De}$ & Oral & $\begin{array}{l}\text { W. } \\
\text { Pongamornkul } \\
3733\end{array}$ \\
\hline \multicolumn{7}{|l|}{ ANACARDIACEAE } \\
\hline Rhus chinensis Mill. & $\begin{array}{l}\text { Saeng- } \\
\text { Chay }\end{array}$ & 0.18 & $\begin{array}{l}\text { Fr: Thirst, } \\
\text { Cough, } \\
\text { Diarrhea } \\
\text { Fr: Laxative, } \\
\text { Stomachache }\end{array}$ & $\begin{array}{l}\text { Np } \\
\text { In }\end{array}$ & $\begin{array}{l}\text { Oral } \\
\text { Oral }\end{array}$ & $\begin{array}{l}\text { W. } \\
\text { Pongamornkul } \\
5145\end{array}$ \\
\hline $\begin{array}{l}\text { Spondias pinnata (L. } \\
\text { f.) Kurz }\end{array}$ & $\begin{array}{l}\text { Phay- } \\
\text { Sae }\end{array}$ & 0.12 & Fr: Cough & $\mathrm{Np}$ & Oral & - \\
\hline \multicolumn{7}{|l|}{ APIACEAE } \\
\hline $\begin{array}{l}\text { Centella asiatica (L.) } \\
\text { Urb. }\end{array}$ & $\begin{array}{l}\text { Thui- } \\
\text { Phu- } \\
\text { Nae- } \\
\text { Du }\end{array}$ & 0.48 & $\begin{array}{l}\text { Wp: } \\
\text { Flatulence, } \\
\text { Cough, } \\
\text { Stomachache } \\
\text { Wp: Muscle } \\
\text { pain } \\
\text { Lf: Apthous } \\
\text { ulcer } \\
\text { Lf: Fever }\end{array}$ & $\begin{array}{l}\mathrm{Np}, \mathrm{De} \\
\mathrm{De} \\
\mathrm{Np} \\
\mathrm{De}\end{array}$ & $\begin{array}{l}\text { Oral } \\
\text { Oral } \\
\text { Oral } \\
\text { Oral }\end{array}$ & $\begin{array}{l}\text { W. } \\
\text { Pongamornkul } \\
4973\end{array}$ \\
\hline Eryngium foetidum L. & $\begin{array}{l}\text { Nang- } \\
\text { Ka- } \\
\text { Bang }\end{array}$ & 0.06 & $\begin{array}{l}\text { Lf: } \\
\text { Stomachache, } \\
\text { Micturate }\end{array}$ & $\mathrm{De}$ & Oral & $\begin{array}{l}\text { W. } \\
\text { Pongamornkul } \\
04045\end{array}$ \\
\hline $\begin{array}{l}\text { Oenanthe javanica } \\
\text { (Blume) DC. }\end{array}$ & $\begin{array}{l}\text { Pha-A- } \\
\text { Du }\end{array}$ & 0.08 & $\begin{array}{l}\text { Lf: Muscle } \\
\text { pain } \\
\text { Wp: } \\
\text { Flatulence, } \\
\text { Stomachache }\end{array}$ & $\begin{array}{l}\mathrm{De} \\
\mathrm{Np}\end{array}$ & $\begin{array}{l}\text { Oral } \\
\text { Oral }\end{array}$ & $\begin{array}{l}\text { W. } \\
\text { Pongamornkul } \\
4547\end{array}$ \\
\hline \multicolumn{7}{|l|}{ AQUIFOLIACEAE } \\
\hline \multicolumn{7}{|c|}{$\begin{array}{l}\text { * Abbreviations for the plant parts: Bk = Bark; Bu = Bulbil; FI = Flower; Fr = Fruit; Ga = Gall; La = Latex; Lf = Leaf; Rh } \\
=\text { Rhizome; Rt = Root; Sd = Seed; St = Stem; Tu = Tuber; Wp = Whole plant }\end{array}$} \\
\hline
\end{tabular}




\begin{tabular}{|lllllll|}
\hline FAMILY & Local & Use & Part used and & Preparation** & Route of & Voucher \\
Scientific names & name & $\begin{array}{l}\text { value } \\
\text { (UV) }\end{array}$ & application* & & administration & number \\
& & & &
\end{tabular}

\begin{tabular}{|c|c|c|c|c|c|c|}
\hline $\begin{array}{l}\text { llex umbellulata } \\
\text { (Wall.) Loes. }\end{array}$ & $\begin{array}{l}\text { Seng- } \\
\text { Je }\end{array}$ & 0.04 & $\begin{array}{l}\text { Fr: } \\
\text { Stomachache }\end{array}$ & $\mathrm{Np}$ & Oral & $\begin{array}{l}\text { W. } \\
\text { Pongamornkul } \\
04274\end{array}$ \\
\hline \multicolumn{7}{|l|}{ ARACEAE } \\
\hline $\begin{array}{l}\text { Pothos chinensis } \\
\text { (Raf.) Merr. }\end{array}$ & $\begin{array}{l}\text { Cha- } \\
\text { Chao- } \\
\text { Mue }\end{array}$ & 0.12 & $\begin{array}{l}\text { Wp: Muscle } \\
\text { pain, } \\
\text { Stomachache } \\
\text { St, Lf: } \\
\text { Diabetes }\end{array}$ & $\begin{array}{l}\text { De } \\
\text { De }\end{array}$ & $\begin{array}{l}\text { Oral } \\
\text { Oral }\end{array}$ & $\begin{array}{l}\text { W. } \\
\text { Pongamornkul } \\
4873\end{array}$ \\
\hline
\end{tabular}

\section{ARALIACEAE}

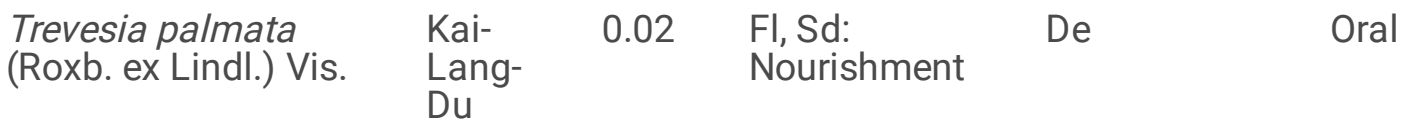

\section{ARECACEAE}

\begin{tabular}{|c|c|c|c|c|c|c|}
\hline $\begin{array}{l}\text { Phoenix loureiroi } \\
\text { Kunth }\end{array}$ & $\begin{array}{l}\text { Ka- } \\
\text { Loe- } \\
\text { Sae }\end{array}$ & 0.02 & Fr: Cough & $\mathrm{Np}$ & Oral & $\begin{array}{l}\text { W. } \\
\text { Pongamornkul } \\
5204\end{array}$ \\
\hline
\end{tabular}

\section{ARISTOLOCHIACEAE}

$\begin{array}{lllll}\begin{array}{l}\text { Aristolochia tagala } \\ \text { Cham. }\end{array} & \begin{array}{l}\text { Ya-Le- } \\ \text { Mu-Du }\end{array} & 0.02 & \text { St, La: Pain } & \text { De }\end{array}$

\section{ASPARAGACEAE}

\begin{tabular}{|c|c|c|c|c|c|c|}
\hline $\begin{array}{l}\text { Asparagus filicinus } \\
\text { Buch.-Ham. ex D. Don }\end{array}$ & $\begin{array}{l}\text { Pho- } \\
\text { Khuai- } \\
\text { Mi }\end{array}$ & 0.32 & $\begin{array}{l}\text { Rt: Muscle } \\
\text { pain, } \\
\text { Stomachache, } \\
\text { Postpartum } \\
\text { period, } \\
\text { Diarrhea, } \\
\text { Stones } \\
\text { Rt: Flatulence }\end{array}$ & $\begin{array}{l}\mathrm{De} \\
\mathrm{De}\end{array}$ & $\begin{array}{l}\text { Oral } \\
\text { Oral }\end{array}$ & $\begin{array}{l}\text { W. } \\
\text { Pongamornkul } \\
4949\end{array}$ \\
\hline
\end{tabular}

\footnotetext{
* Abbreviations for the plant parts: $\mathrm{Bk}=$ Bark; $\mathrm{Bu}=$ Bulbil; FI = Flower; Fr = Fruit; $\mathrm{Ga}=\mathrm{Gall} ; \mathrm{La}=\mathrm{Latex} ; \mathrm{Lf}=\mathrm{Leaf} ; \mathrm{Rh}$

$=$ Rhizome; Rt = Root; Sd = Seed; St = Stem; Tu = Tuber; $\mathrm{Wp}=$ Whole plant

**Abbreviations for the preparation methods: $\mathrm{Bu}=$ Burned; $\mathrm{De}=$ Decoction; $\mathrm{In}=$ Infusion; $\mathrm{Np}=$ Non-prepared ; Po

$=$ Pounded
} 


\begin{tabular}{|c|c|c|c|c|c|c|}
\hline $\begin{array}{l}\text { FAMILY } \\
\text { Scientific names }\end{array}$ & $\begin{array}{l}\text { Local } \\
\text { name }\end{array}$ & $\begin{array}{l}\text { Use } \\
\text { value } \\
\text { (UV) }\end{array}$ & $\begin{array}{l}\text { Part used and } \\
\text { application* }\end{array}$ & Preparation** & $\begin{array}{l}\text { Route of } \\
\text { administration }\end{array}$ & $\begin{array}{l}\text { Voucher } \\
\text { number }\end{array}$ \\
\hline $\begin{array}{l}\text { Tupistra muricata } \\
\text { (Gagnep.) N. Tanaka }\end{array}$ & $\begin{array}{l}\text { Ta-Si- } \\
\text { Khe-La }\end{array}$ & 0.16 & $\begin{array}{l}\text { Lf: Bruised, } \\
\text { Pain }\end{array}$ & $\mathrm{Bu}$ & $\begin{array}{l}\text { Poultices } \\
\text { Poultices }\end{array}$ & $\begin{array}{l}\text { W. } \\
\text { Pongamornkul } \\
3470\end{array}$ \\
\hline \multicolumn{7}{|l|}{ ASTERACEAE } \\
\hline $\begin{array}{l}\text { Acmella paniculata } \\
\text { (Wall. ex DC.) R. K. } \\
\text { Jansen }\end{array}$ & $\begin{array}{l}\text { Wan- } \\
\text { Than- } \\
\text { Du }\end{array}$ & 0.06 & $\begin{array}{l}\text { Lf: Diarrhea } \\
\text { Wp: Dental } \\
\text { caries } \\
\text { Lf, Fl: } \\
\text { Anthelmintic }\end{array}$ & $\begin{array}{l}\mathrm{De} \\
\mathrm{Np} \\
\mathrm{Np}\end{array}$ & $\begin{array}{l}\text { Oral } \\
\text { Oral } \\
\text { Oral }\end{array}$ & $\begin{array}{l}\text { W. } \\
\text { Pongamornkul } \\
4954\end{array}$ \\
\hline $\begin{array}{l}\text { Ageratina } \\
\text { adenophora } \\
\text { (Spreng.) R. M. King } \\
\text { \& H. Rob. }\end{array}$ & $\begin{array}{l}\text { Nang- } \\
\text { Ka- } \\
\text { Bang }\end{array}$ & 0.94 & $\begin{array}{l}\text { Lf: Anti- } \\
\text { bleeding, } \\
\text { Wounds }\end{array}$ & Po & Poultices & $\begin{array}{l}\text { W. } \\
\text { Pongamornkul } \\
3748\end{array}$ \\
\hline $\begin{array}{l}\text { Blumea balsamifera } \\
\text { (L.) DC. }\end{array}$ & $\begin{array}{l}\text { Pho- } \\
\text { Nae }\end{array}$ & 0.92 & $\begin{array}{l}\text { Lf: } \\
\text { Postpartum } \\
\text { period } \\
\text { Wp: Muscle } \\
\text { pain } \\
\text { Lf: Wounds }\end{array}$ & $\begin{array}{l}\mathrm{Bu} \\
\mathrm{Bu} \\
\mathrm{Po}\end{array}$ & $\begin{array}{l}\text { Poultices } \\
\text { Poultices } \\
\text { Poultices }\end{array}$ & $\begin{array}{l}\text { W. } \\
\text { Pongamornkul } \\
3750\end{array}$ \\
\hline $\begin{array}{l}\text { Chromolaena } \\
\text { odorata (L.) R. M. } \\
\text { King \& H. Rob. }\end{array}$ & $\begin{array}{l}\text { Cho- } \\
\text { Pho- } \\
\text { Ko }\end{array}$ & 0.88 & $\begin{array}{l}\text { Rt: } \\
\text { Stomachache, } \\
\text { Flatulence } \\
\text { Lf: Anti- } \\
\text { bleeding, } \\
\text { Wounds }\end{array}$ & $\begin{array}{l}\text { De } \\
\text { Po }\end{array}$ & $\begin{array}{l}\text { Oral } \\
\text { Poultices }\end{array}$ & $\begin{array}{l}\text { W. } \\
\text { Pongamornkul } \\
3751\end{array}$ \\
\hline $\begin{array}{l}\text { Crassocephalum } \\
\text { crepidioides (Benth.) } \\
\text { S. Moore }\end{array}$ & $\begin{array}{l}\text { Ta-Bo- } \\
\text { Du }\end{array}$ & 0.04 & Lf: Flatulence & $\mathrm{De}$ & Oral & $\begin{array}{l}\text { W. } \\
\text { Pongamornkul } \\
3753\end{array}$ \\
\hline $\begin{array}{l}\text { Decaneuropsis cf. } \\
\text { eberhardtii (Gagnep.) } \\
\text { H. Rob. \& Skvarla }\end{array}$ & Ya-Ke & 0.42 & $\begin{array}{l}\text { St: Appetizing, } \\
\text { Muscle pain, } \\
\text { Diabetes } \\
\text { Wp: Pain, } \\
\text { Drunk } \\
\text { St: Cough }\end{array}$ & $\begin{array}{l}\mathrm{De} \\
\mathrm{De} \\
\mathrm{Np}\end{array}$ & $\begin{array}{l}\text { Oral } \\
\text { Oral } \\
\text { Oral }\end{array}$ & - \\
\hline $\begin{array}{l}\text { Elephantopus scaber } \\
\text { L. }\end{array}$ & $\begin{array}{l}\text { Ta-Chi- } \\
\text { Ko }\end{array}$ & 0.72 & $\begin{array}{l}\text { Rt: Cough, } \\
\text { Bruised, } \\
\text { Insomnia } \\
\text { Rt: } \\
\text { Nourishment } \\
\text { Rt, St, Lf: } \\
\text { Fever }\end{array}$ & $\begin{array}{l}\mathrm{De} \\
\mathrm{De} \\
\mathrm{De}\end{array}$ & $\begin{array}{l}\text { Oral } \\
\text { Bath } \\
\text { Oral }\end{array}$ & $\begin{array}{l}\text { W. } \\
\text { Pongamornkul } \\
5180\end{array}$ \\
\hline
\end{tabular}

\footnotetext{
* Abbreviations for the plant parts: Bk = Bark; Bu = Bulbil; FI = Flower; Fr = Fruit; Ga = Gall; La = Latex; Lf = Leaf; Rh

$=$ Rhizome; Rt = Root; Sd = Seed; St = Stem; Tu = Tuber; $\mathrm{Wp}=$ Whole plant

**Abbreviations for the preparation methods: $\mathrm{Bu}=$ Burned; $\mathrm{De}=$ Decoction; $\mathrm{In}=$ Infusion; $\mathrm{Np}=$ Non-prepared ; Po $=$ Pounded
} 


\begin{tabular}{|c|c|c|c|c|c|c|}
\hline $\begin{array}{l}\text { FAMILY } \\
\text { Scientific names }\end{array}$ & $\begin{array}{l}\text { Local } \\
\text { name }\end{array}$ & $\begin{array}{l}\text { Use } \\
\text { value } \\
\text { (UV) }\end{array}$ & $\begin{array}{l}\text { Part used and } \\
\text { application* }\end{array}$ & Preparation** & $\begin{array}{l}\text { Route of } \\
\text { administration }\end{array}$ & $\begin{array}{l}\text { Voucher } \\
\text { number }\end{array}$ \\
\hline $\begin{array}{l}\text { Gerbera piloselloides } \\
\text { (L.) Cass. }\end{array}$ & $\begin{array}{l}\text { Ta-Chi- } \\
\text { Ko }\end{array}$ & 0.4 & $\begin{array}{l}\text { Wp: Cough } \\
\text { Rt: Insomnia, } \\
\text { Bruised, } \\
\text { Nourishment }\end{array}$ & $\begin{array}{l}\mathrm{De} \\
\mathrm{De}\end{array}$ & $\begin{array}{l}\text { Oral } \\
\text { Oral }\end{array}$ & $\begin{array}{l}\text { W. } \\
\text { Pongamornkul } \\
5263\end{array}$ \\
\hline $\begin{array}{l}\text { Microglossa pyrifolia } \\
\text { (Lam.) Kuntze }\end{array}$ & $\begin{array}{l}\text { Pho- } \\
\text { Sa- } \\
\text { Seng }\end{array}$ & 0.04 & $\begin{array}{l}\text { Lf: Fever } \\
\text { Lf: Bruised }\end{array}$ & $\begin{array}{l}\mathrm{De} \\
\mathrm{Bu}\end{array}$ & $\begin{array}{l}\text { Bath } \\
\text { Liniment }\end{array}$ & $\begin{array}{l}\text { W. } \\
\text { Pongamornkul } \\
5612\end{array}$ \\
\hline Tagetes erecta L. & $\begin{array}{l}\text { Pho- } \\
\text { Pho }\end{array}$ & 0.02 & St: Diarrhea & $\mathrm{De}$ & Oral & $\begin{array}{l}\text { W. } \\
\text { Pongamornkul } \\
5234\end{array}$ \\
\hline \multicolumn{7}{|l|}{ BASELLACEAE } \\
\hline $\begin{array}{l}\text { Anredera cordifolia } \\
\text { (Ten.) Steenis }\end{array}$ & $\begin{array}{l}\text { Ta- } \\
\text { Phe- } \\
\text { La- } \\
\text { Chae- } \\
\text { Wa }\end{array}$ & 0.12 & $\begin{array}{l}\text { Bu: Flame } \\
\text { burn } \\
\text { Bu: Urinate, } \\
\text { Bu: } \\
\text { Nourishment } \\
\text { Fr: Poison } \\
\text { Bu: } \\
\text { Contraception }\end{array}$ & $\begin{array}{l}\mathrm{Po} \\
\mathrm{De} \\
\mathrm{Np} \\
\mathrm{Np} \\
\mathrm{Np}\end{array}$ & $\begin{array}{l}\text { Liniment } \\
\text { Oral } \\
\text { Oral } \\
\text { Oral } \\
\text { Oral }\end{array}$ & $\begin{array}{l}\text { W. } \\
\text { Pongamornkul } \\
4560\end{array}$ \\
\hline \multicolumn{7}{|l|}{ BEGONIACEAE } \\
\hline Begonia sp. & $\begin{array}{l}\text { Ta- } \\
\text { Chay- } \\
\text { Ko }\end{array}$ & 0.12 & Wp: Itching & Po & Poultices & $\begin{array}{l}\text { W. } \\
\text { Pongamornkul } \\
4759\end{array}$ \\
\hline \multicolumn{7}{|l|}{ BETULACEAE } \\
\hline $\begin{array}{l}\text { Betula alnoides } \\
\text { Buch.-Ham. ex G. } \\
\text { Don }\end{array}$ & $\begin{array}{l}\text { Mai- } \\
\text { Mi- } \\
\text { Kang }\end{array}$ & 0.58 & $\begin{array}{l}\text { St: } \\
\text { Nourishment } \\
\text { Bk: Muscle } \\
\text { pain, Cough, } \\
\text { Gastritis, } \\
\text { Lassitude, } \\
\text { Blood } \\
\text { circulation }\end{array}$ & $\begin{array}{l}\text { De } \\
\text { De }\end{array}$ & $\begin{array}{l}\text { Oral } \\
\text { Oral }\end{array}$ & $\begin{array}{l}\text { W. } \\
\text { Pongamornkul } \\
4724\end{array}$ \\
\hline \multicolumn{7}{|l|}{ BIGNONIACEAE } \\
\hline $\begin{array}{l}\text { Oroxylum indicum } \\
\text { (L.) Kurz }\end{array}$ & $\begin{array}{l}\text { Du- } \\
\text { Kae- } \\
\text { Sae }\end{array}$ & 0.04 & $\begin{array}{l}\text { St: Anti-HIV } \\
\text { FI, Fr: Muscle } \\
\text { pain }\end{array}$ & $\begin{array}{l}\mathrm{De} \\
\mathrm{Np}\end{array}$ & $\begin{array}{l}\text { Oral } \\
\text { Oral }\end{array}$ & $\begin{array}{l}\text { W. } \\
\text { Pongamornkul } \\
454\end{array}$ \\
\hline \multicolumn{7}{|l|}{ BURSERACEAE } \\
\hline $\begin{array}{l}\text { Protium serratum } \\
\text { (Wall. ex Colebr.) } \\
\text { Engl. }\end{array}$ & $\begin{array}{l}\text { Phi- } \\
\text { Sae }\end{array}$ & 0.02 & Fr: Vomiting & $\mathrm{Np}$ & Oral & $\begin{array}{l}\text { W. } \\
\text { Pongamornkul } \\
04238\end{array}$ \\
\hline
\end{tabular}

\footnotetext{
* Abbreviations for the plant parts: Bk = Bark; Bu = Bulbil; FI = Flower; Fr = Fruit; Ga = Gall; La = Latex; Lf = Leaf; Rh $=$ Rhizome; Rt = Root; Sd = Seed; St = Stem; Tu = Tuber; $\mathrm{Wp}=$ Whole plant

**Abbreviations for the preparation methods: $\mathrm{Bu}=$ Burned; $\mathrm{De}=$ Decoction; $\mathrm{In}=$ Infusion; $\mathrm{Np}=$ Non-prepared ; Po $=$ Pounded
} 


\begin{tabular}{|lllllll}
\hline FAMILY & Local & Use & Part used and & Preparation** & Route of & Voucher \\
Scientific names & name & value & application* & & administration & number \\
& & (UV) & & &
\end{tabular}

\section{CELASTRACEAE}

\begin{tabular}{|c|c|c|c|c|c|c|}
\hline $\begin{array}{l}\text { Celastrus paniculatus } \\
\text { Willd. }\end{array}$ & $\begin{array}{l}\text { Ble- } \\
\text { Bang- } \\
\text { Yo }\end{array}$ & 0.02 & Rt: Dysuria & $\mathrm{De}$ & Oral & $\begin{array}{l}\text { W. } \\
\text { Pongamornkul } \\
\text { et al. } 5088\end{array}$ \\
\hline
\end{tabular}

\section{CLUSIACEAE}

Garcinia cf. pedunculata Roxb. ex Kuy-Sa

Ko- $\quad 0.76$

Buch.-Ham.
Fr: Diabetes,
Cancer,
Flatulence
Fr: Drunk,
Stones,
Hypertension,
Headache,
Lumbago,
Diarrhea
Fr: Insect
bites

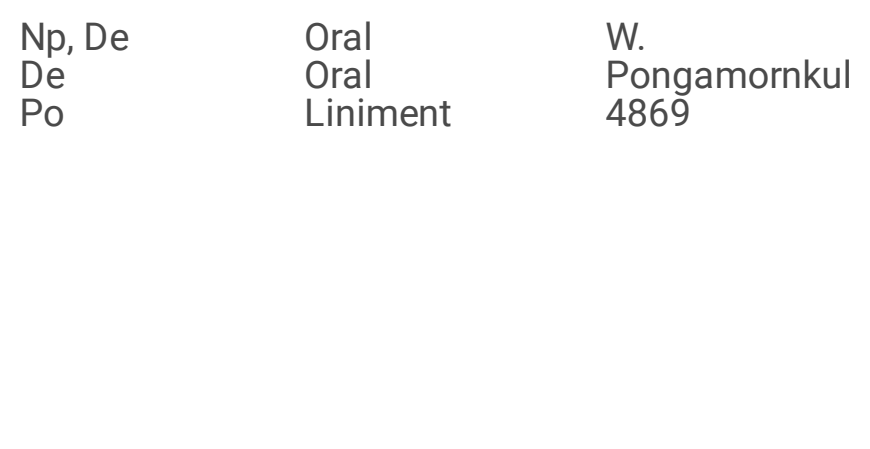

Np, De

Do

Oral

Pongamornkul 4869

\section{COMBRETACEAE}

\begin{tabular}{|c|c|c|c|c|c|c|}
\hline $\begin{array}{l}\text { Anogeissus } \\
\text { acuminata (Roxb. ex } \\
\text { DC.) Wall. ex Guillem. } \\
\text { \& Perr. }\end{array}$ & $\begin{array}{l}\text { Seng- } \\
\text { Cha- } \\
\text { Kad }\end{array}$ & 0.02 & Rt: Dizziness & $\mathrm{De}$ & Oral & $\begin{array}{l}\text { W. } \\
\text { Pongamornkul } \\
4694\end{array}$ \\
\hline $\begin{array}{l}\text { Terminalia chebula } \\
\text { Retz. }\end{array}$ & $\begin{array}{l}\text { Ma- } \\
\text { Nae- } \\
\text { Sae }\end{array}$ & 0.1 & Fr: Cough & $\mathrm{Np}$ & Oral & $\begin{array}{l}\text { W. } \\
\text { Pongamornkul } \\
5215\end{array}$ \\
\hline
\end{tabular}

\section{COMMELINACEAE}

\begin{tabular}{|c|c|c|c|c|c|c|}
\hline $\begin{array}{l}\text { Commelina diffusa } \\
\text { Burm.f. }\end{array}$ & $\begin{array}{l}\text { Phu- } \\
\text { Du }\end{array}$ & 0.02 & Lf: Cough & $\mathrm{Np}$ & oral & $\begin{array}{l}\text { W. } \\
\text { Pongamornkul } \\
4712\end{array}$ \\
\hline
\end{tabular}

\section{COSTACEAE}

\begin{tabular}{|c|c|c|c|c|c|c|}
\hline $\begin{array}{l}\text { Hellenia speciosa (J. } \\
\text { Koenig) S. R. Dutta } \\
\text { Specht }\end{array}$ & Su-Lay & 0.08 & St: Snake bite & Po & Liniment & $\begin{array}{l}\text { W. } \\
\text { Pongamornkul } \\
3237\end{array}$ \\
\hline \multicolumn{7}{|l|}{ CUCURBITACEAE } \\
\hline $\begin{array}{l}\text { Gynostemma } \\
\text { pentaphyllum } \\
\text { (Thunb.) Makino }\end{array}$ & $\begin{array}{l}\text { Tu- } \\
\text { Kha- } \\
\text { Du }\end{array}$ & 0.02 & Lf: Diabetes & $\mathrm{De}$ & Oral & $\begin{array}{l}\text { W. } \\
\text { Pongamornkul } \\
3437\end{array}$ \\
\hline $\begin{array}{l}\text { Momordica charantia } \\
\text { L. }\end{array}$ & $\begin{array}{l}\text { Mang- } \\
\text { Ka-Lae }\end{array}$ & 0.02 & $\begin{array}{l}\text { Lf, fr: Muscle } \\
\text { pain }\end{array}$ & $\mathrm{De}$ & Oral & - \\
\hline
\end{tabular}

\footnotetext{
* Abbreviations for the plant parts: Bk = Bark; Bu = Bulbil; FI = Flower; Fr = Fruit; Ga = Gall; La = Latex; Lf = Leaf; Rh $=$ Rhizome; Rt = Root; Sd = Seed; St = Stem; Tu = Tuber; Wp = Whole plant

**Abbreviations for the preparation methods: Bu = Burned; De = Decoction; In = Infusion; Np = Non-prepared ; Po $=$ Pounded
} 


\begin{tabular}{|lllllll}
\hline FAMILY & Local & Use & Part used and & Preparation** & Route of & Voucher \\
Scientific names & name & value & application* & & administration & number \\
& & (UV) & & &
\end{tabular}

\section{DAVALLIACEAE}

Davallia sp.

Ta-Si- $\quad 0.06$

St: Snake bite

St: Diuretic

Po

Liniment

W.

Pongamornkul 5020

\section{EQUISETACEAE}

\begin{tabular}{|c|c|c|c|c|c|c|}
\hline $\begin{array}{l}\text { Hippochaete debilis } \\
\text { (Roxb. ex Vaucher) } \\
\text { Ching }\end{array}$ & $\begin{array}{l}\text { No-Ka- } \\
\text { Chi- } \\
\text { Sae }\end{array}$ & 0.16 & $\begin{array}{l}\text { Wp: Muscle } \\
\text { pain, Stones, } \\
\text { Cough, } \\
\text { Stomachache }\end{array}$ & $\mathrm{De}$ & Oral & $\begin{array}{l}\text { W. } \\
\text { Pongamornku } \\
5231\end{array}$ \\
\hline
\end{tabular}

\section{EUPHORBIACEAE}

\begin{tabular}{|c|c|c|c|c|c|c|}
\hline $\begin{array}{l}\text { Antidesma acidum } \\
\text { Retz. }\end{array}$ & $\begin{array}{l}\text { Nang- } \\
\text { Sa-Sai }\end{array}$ & 0.02 & Lf: Pain & $\mathrm{De}$ & Bath & $\begin{array}{l}\text { W. } \\
\text { Pongamornkul } \\
3782\end{array}$ \\
\hline $\begin{array}{l}\text { Balakata baccata } \\
\text { (Roxb.) Esser }\end{array}$ & Ta-Sae & 0.02 & Lf: Laxative & $\mathrm{De}$ & Oral & $\begin{array}{l}\text { W. } \\
\text { Pongamornkul } \\
4887\end{array}$ \\
\hline $\begin{array}{l}\text { Falconeria insignis } \\
\text { Royle }\end{array}$ & $\begin{array}{l}\text { Se-Blu- } \\
\text { Mi }\end{array}$ & 0.12 & $\begin{array}{l}\text { La: Abscess, } \\
\text { Wounds and } \\
\text { Boils }\end{array}$ & $\mathrm{Np}$ & Liniment & $\begin{array}{l}\text { W. } \\
\text { Pongamornkul } \\
5290\end{array}$ \\
\hline $\begin{array}{l}\text { Flueggea virosa } \\
\text { (Roxb. ex Willd.) } \\
\text { Voigt }\end{array}$ & $\begin{array}{l}\text { Seng- } \\
\text { Chang- } \\
\text { Chao }\end{array}$ & 0.06 & $\begin{array}{l}\text { Lf: } \\
\text { Postpartum } \\
\text { blood } \\
\text { circulation } \\
\text { Rt: } \\
\text { Stomachache } \\
\text { Wp: Muscle } \\
\text { pain }\end{array}$ & $\begin{array}{l}\text { De } \\
\text { De } \\
\text { De }\end{array}$ & $\begin{array}{l}\text { Oral } \\
\text { Oral } \\
\text { Oral }\end{array}$ & $\begin{array}{l}\text { W. } \\
\text { Pongamornkul } \\
04197\end{array}$ \\
\hline $\begin{array}{l}\text { Glochidion } \\
\text { sphaerogynum (Müll. } \\
\text { Arg.) Kurz }\end{array}$ & $\begin{array}{l}\text { Pha- } \\
\text { Phea- } \\
\text { Chay }\end{array}$ & 0.02 & $\begin{array}{l}\text { Lf: } \\
\text { Postpartum } \\
\text { blood } \\
\text { circulation }\end{array}$ & $\mathrm{De}$ & Oral & $\begin{array}{l}\text { W. } \\
\text { Pongamornkul } \\
4824\end{array}$ \\
\hline
\end{tabular}

\section{FABACEAE}

$\begin{array}{lllllll}\begin{array}{l}\text { Senegalia rugata } \\ \text { (Lam.) Britton \& Rose }\end{array} & \begin{array}{l}\text { Phu } \\ \text { Che Sa }\end{array} & 0.02 & \text { Fr: Alopecia } & \text { De } & \text { Bath } & \begin{array}{l}\text { W. } \\ \text { Pongamornkul } \\ 4846\end{array} \\ \begin{array}{l}\text { Archidendron } \\ \begin{array}{l}\text { clypearia (Jack) I. C. } \\ \text { Nielsen }\end{array}\end{array} & \begin{array}{l}\text { Cha- } \\ \text { Mi- } \\ \text { Thao }\end{array} & 0.02 & \begin{array}{l}\text { Rt: } \\ \text { Themachache }\end{array} & \text { De } & \text { Oral } & \begin{array}{l}\text { W. } \\ \text { Pongamornkul } \\ 4593\end{array}\end{array}$

\footnotetext{
* Abbreviations for the plant parts: Bk = Bark; Bu= Bulbil; FI = Flower; Fr = Fruit; Ga = Gall; La = Latex; Lf = Leaf; Rh $=$ Rhizome; Rt = Root; Sd = Seed; St = Stem; Tu = Tuber; $\mathrm{Wp}=$ Whole plant

**Abbreviations for the preparation methods: Bu = Burned; De = Decoction; In = Infusion; Np = Non-prepared ; Po $=$ Pounded
} 


\begin{tabular}{|c|c|c|c|c|c|c|}
\hline $\begin{array}{l}\text { FAMILY } \\
\text { Scientific names }\end{array}$ & $\begin{array}{l}\text { Local } \\
\text { name }\end{array}$ & $\begin{array}{l}\text { Use } \\
\text { value } \\
\text { (UV) }\end{array}$ & $\begin{array}{l}\text { Part used and } \\
\text { application* }\end{array}$ & Preparation** & $\begin{array}{l}\text { Route of } \\
\text { administration }\end{array}$ & $\begin{array}{l}\text { Voucher } \\
\text { number }\end{array}$ \\
\hline Bauhinia sp. & $\begin{array}{l}\text { Pa-Na- } \\
\text { Kho- } \\
\text { Thao }\end{array}$ & 0.02 & $\begin{array}{l}\text { Lf, Bk: } \\
\text { Wounds }\end{array}$ & $\mathrm{De}$ & Oral & $\begin{array}{l}\text { W. } \\
\text { Pongamornkul } \\
4612\end{array}$ \\
\hline Dalbergiasp. & Sa-Klo & 0.02 & Rt: Cough & $\mathrm{De}$ & Oral & $\begin{array}{l}\text { W. } \\
\text { Pongamornkul } \\
3807\end{array}$ \\
\hline $\begin{array}{l}\text { Millettia caerulea } \\
\text { Baker }\end{array}$ & $\begin{array}{l}\text { Se-Na- } \\
0\end{array}$ & 0.2 & $\begin{array}{l}\text { Rt: Diarrhea } \\
\text { Lf: } \\
\text { Stomachache, } \\
\text { Flatulence, } \\
\text { Stones }\end{array}$ & $\begin{array}{l}\mathrm{De} \\
\mathrm{De}\end{array}$ & $\begin{array}{l}\text { Oral } \\
\text { Oral }\end{array}$ & $\begin{array}{l}\text { W. } \\
\text { Pongamornkul } \\
5338\end{array}$ \\
\hline Mimosa pudica L. & $\begin{array}{l}\text { Mi-Ta- } \\
\text { Si }\end{array}$ & 0.36 & $\begin{array}{l}\text { Wp: Muscle } \\
\text { pain, Easy } \\
\text { birth, } \\
\text { Stomachache, } \\
\text { Cough, } \\
\text { Anthelmintic, } \\
\text { Bleary-eyed, } \\
\text { Insomnia } \\
\text { Wp: Itching } \\
\text { Rt: Fever } \\
\text { Rt: Dizziness } \\
\text { Rt, St, Lf: } \\
\text { Stones }\end{array}$ & $\begin{array}{l}\text { De } \\
\text { In } \\
\text { In } \\
\text { De } \\
\text { De }\end{array}$ & $\begin{array}{l}\text { Oral } \\
\text { Bath } \\
\text { Bath } \\
\text { Oral } \\
\text { Oral }\end{array}$ & $\begin{array}{l}\text { W. } \\
\text { Pongamornkul } \\
3802\end{array}$ \\
\hline $\begin{array}{l}\text { Tadehagi triquetrum } \\
\text { (L.) H. Ohashi }\end{array}$ & $\begin{array}{l}\text { Ta-Si- } \\
\text { Cho- } \\
\text { Bel }\end{array}$ & 0.34 & $\begin{array}{l}\text { Rt: Muscle } \\
\text { pain, } \\
\text { Stomachache } \\
\text { Lf: Asthma } \\
\text { St: Stones } \\
\text { Wp: Tonic, } \\
\text { Anthelmintic } \\
\text { Rt, St, Lf: } \\
\text { Fever }\end{array}$ & $\begin{array}{l}\mathrm{De} \\
\mathrm{De} \\
\text { In } \\
\mathrm{De} \\
\mathrm{De}\end{array}$ & $\begin{array}{l}\text { Oral } \\
\text { Oral } \\
\text { Oral } \\
\text { Oral } \\
\text { Oral }\end{array}$ & $\begin{array}{l}\text { W. } \\
\text { Pongamornkul } \\
4959\end{array}$ \\
\hline Tamarindus indica L. & $\begin{array}{l}\text { Ma- } \\
\text { Khae- } \\
\text { Sae }\end{array}$ & 0.04 & $\begin{array}{l}\text { Fr: Dizziness } \\
\text { Bk: Cough }\end{array}$ & $\begin{array}{l}\mathrm{Np} \\
\mathrm{Np}\end{array}$ & $\begin{array}{l}\text { Oral } \\
\text { Oral }\end{array}$ & $\begin{array}{l}\text { W. } \\
\text { Pongamornkul } \\
3805\end{array}$ \\
\hline $\begin{array}{l}\text { Xylia xylocarpa } \\
\text { (Roxb.) W. Theob. }\end{array}$ & $\begin{array}{l}\text { Khwai- } \\
\text { Sae }\end{array}$ & 0.04 & $\begin{array}{l}\text { St, Lf: Blood } \\
\text { tonic } \\
\text { Fr: Cough }\end{array}$ & $\begin{array}{l}\mathrm{De} \\
\mathrm{De}\end{array}$ & $\begin{array}{l}\text { Oral } \\
\text { Oral }\end{array}$ & $\begin{array}{l}\text { W. } \\
\text { Pongamornkul } \\
4692\end{array}$ \\
\hline \multicolumn{7}{|l|}{ FAGACEAE } \\
\hline Lithocarpus sp. & Si-Phai & 0.04 & $\begin{array}{l}\text { Bk: Cough } \\
\text { St: } \\
\text { Hypertension }\end{array}$ & $\begin{array}{l}\mathrm{Np} \\
\mathrm{De}\end{array}$ & $\begin{array}{l}\text { Oral } \\
\text { Oral }\end{array}$ & - \\
\hline \multicolumn{7}{|l|}{ GENTIANACEAE } \\
\hline \multicolumn{7}{|c|}{$\begin{array}{l}\text { * Abbreviations for the plant parts: Bk = Bark; Bu = Bulbil; FI = Flower; Fr = Fruit; Ga = Gall; La = Latex; Lf = Leaf; Rh } \\
\text { = Rhizome; Rt = Root; Sd = Seed; St = Stem; Tu = Tuber; Wp = Whole plant }\end{array}$} \\
\hline
\end{tabular}




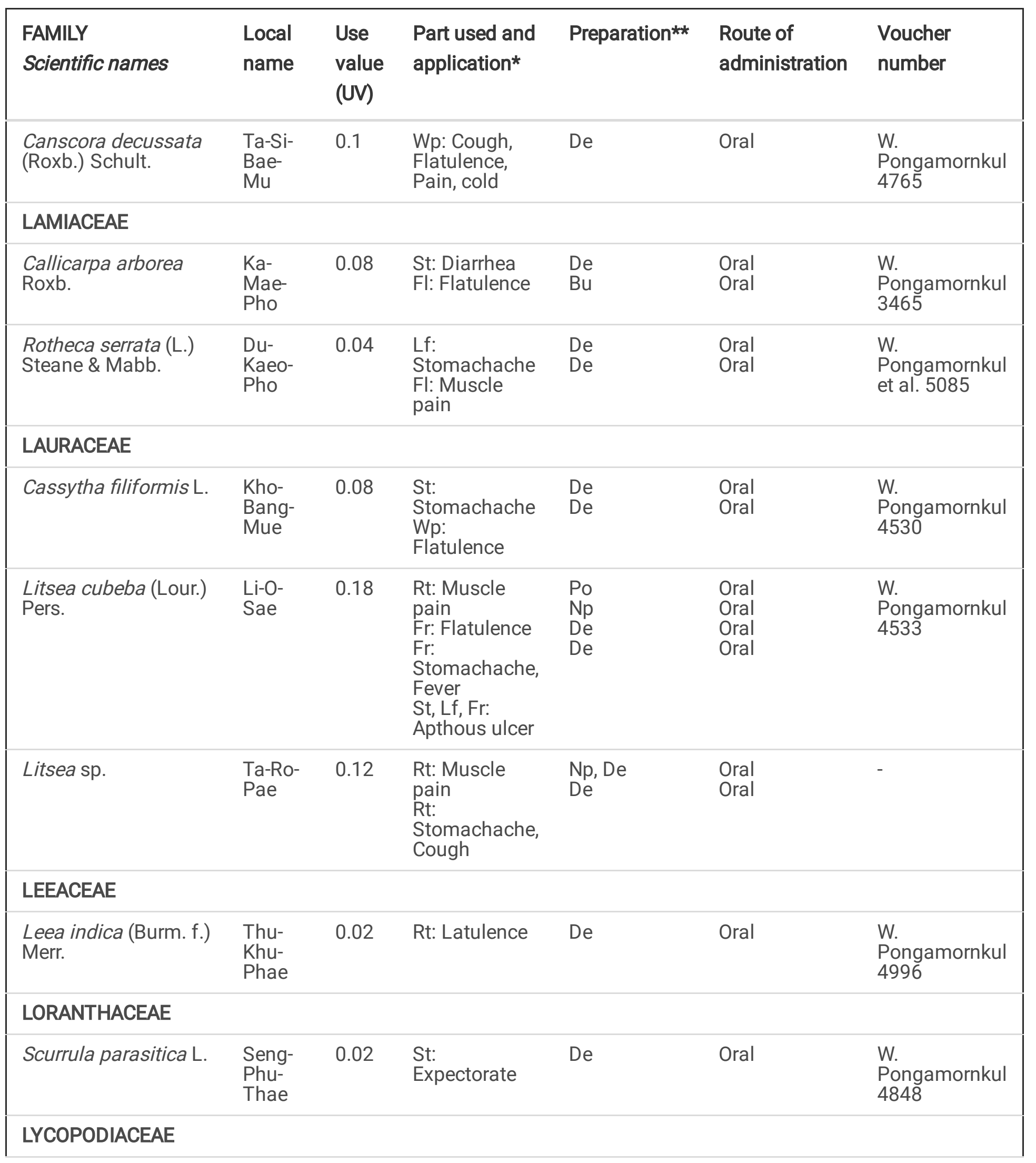

\footnotetext{
* Abbreviations for the plant parts: Bk = Bark; Bu = Bulbil; FI = Flower; Fr = Fruit; Ga = Gall; La = Latex; Lf = Leaf; Rh $=$ Rhizome; Rt = Root; Sd = Seed; St = Stem; Tu = Tuber; Wp = Whole plant

**Abbreviations for the preparation methods: Bu = Burned; De = Decoction; In = Infusion; Np = Non-prepared ; Po $=$ Pounded
} 


\begin{tabular}{|c|c|c|c|c|c|c|}
\hline $\begin{array}{l}\text { FAMILY } \\
\text { Scientific names }\end{array}$ & $\begin{array}{l}\text { Local } \\
\text { name }\end{array}$ & $\begin{array}{l}\text { Use } \\
\text { value } \\
\text { (UV) }\end{array}$ & $\begin{array}{l}\text { Part used and } \\
\text { application* }\end{array}$ & Preparation** & $\begin{array}{l}\text { Route of } \\
\text { administration }\end{array}$ & $\begin{array}{l}\text { Voucher } \\
\text { number }\end{array}$ \\
\hline $\begin{array}{l}\text { Lycopodium } \\
\text { clavatum L. }\end{array}$ & $\begin{array}{l}\text { Ta-Si- } \\
\text { Lae- } \\
\text { Khae }\end{array}$ & 0.04 & $\begin{array}{l}\text { Wp: Muscle } \\
\text { pain }\end{array}$ & $\mathrm{De}$ & Oral & - \\
\hline \multicolumn{7}{|l|}{ LYTHRACEAE } \\
\hline $\begin{array}{l}\text { Duabanga } \\
\text { grandiflora (DC.) } \\
\text { Walp. }\end{array}$ & $\mathrm{Ku}$ & 0.02 & Lf: Dry skin & Po & Poultices & $\begin{array}{l}\text { W. } \\
\text { Pongamornkul } \\
5626\end{array}$ \\
\hline \multicolumn{7}{|l|}{ MALVACEAE } \\
\hline Sida acuta Burm. f. & $\begin{array}{l}\text { Nang- } \\
\text { Khu-Mi }\end{array}$ & 0.04 & Lf: Wounds & Po & Poultices & $\begin{array}{l}\text { W. } \\
\text { Pongamornkul } \\
4485\end{array}$ \\
\hline \multicolumn{7}{|l|}{ MELANTHIACEAE } \\
\hline $\begin{array}{l}\text { Daiswa polyphylla } \\
\text { (Sm.) Raf. }\end{array}$ & Ta-Thi & 0.16 & $\begin{array}{l}\text { Tu: Gastritis, } \\
\text { Backache, } \\
\text { Cancer, } \\
\text { Nourishment, } \\
\text { Pain }\end{array}$ & $\mathrm{De}$ & Oral & $\begin{array}{l}\text { W. } \\
\text { Pongamornkul } \\
5321\end{array}$ \\
\hline \multicolumn{7}{|l|}{ MELASTOMATACEAE } \\
\hline $\begin{array}{l}\text { Melastoma } \\
\text { malabathricum L. } \\
\text { subsp. } \\
\text { malabathricum }\end{array}$ & $\begin{array}{l}\text { Se-Sa- } \\
\text { Phli }\end{array}$ & 0.06 & $\begin{array}{l}\text { Rt: Cough } \\
\text { Rt, St: Stones }\end{array}$ & $\begin{array}{l}\mathrm{De} \\
\mathrm{De}\end{array}$ & $\begin{array}{l}\text { Oral } \\
\text { Oral }\end{array}$ & $\begin{array}{l}\text { W. } \\
\text { Pongamornkul } \\
4756\end{array}$ \\
\hline \multicolumn{7}{|l|}{ MENIAPERMACEAE } \\
\hline $\begin{array}{l}\text { Tinospora crispa (L.) } \\
\text { Hook. f. \& Thomson }\end{array}$ & $\begin{array}{l}\text { Seng- } \\
\text { Khi-Mi }\end{array}$ & 0.1 & $\begin{array}{l}\text { St: Muscle } \\
\text { pain, Cough } \\
\text { St: } \\
\text { Nourishment } \\
\text { St: Pain } \\
\text { La: Rashes }\end{array}$ & $\begin{array}{l}\mathrm{De} \\
\mathrm{In} \\
\mathrm{Np} \\
\mathrm{Np}\end{array}$ & $\begin{array}{l}\text { Oral } \\
\text { Oral } \\
\text { Oral } \\
\text { Liniment }\end{array}$ & $\begin{array}{l}\text { W. } \\
\text { Pongamornkul } \\
03931\end{array}$ \\
\hline \multicolumn{7}{|l|}{ MORACEAE } \\
\hline $\begin{array}{l}\text { Artocarpus } \\
\text { heterophyllus Lam. }\end{array}$ & $\begin{array}{l}\text { Pa- } \\
\text { Nuai- } \\
\text { Sae }\end{array}$ & 0.02 & Fr: Cough & $\mathrm{Np}$ & Oral & \\
\hline Ficus hispida L. f. & $\begin{array}{l}\text { A-Nae- } \\
\text { Sae }\end{array}$ & 0.02 & La: Abscess & $\mathrm{Np}$ & Liniment & $\begin{array}{l}\text { W. } \\
\text { Pongamornkul } \\
4619\end{array}$ \\
\hline
\end{tabular}

\footnotetext{
* Abbreviations for the plant parts: Bk = Bark; Bu = Bulbil; FI = Flower; Fr = Fruit; Ga = Gall; La = Latex; Lf = Leaf; Rh $=$ Rhizome; Rt = Root; Sd = Seed; St = Stem; Tu = Tuber; $\mathrm{Wp}=$ Whole plant

**Abbreviations for the preparation methods: Bu = Burned; De = Decoction; In = Infusion; Np = Non-prepared ; Po $=$ Pounded
} 


\begin{tabular}{|c|c|c|c|c|c|c|}
\hline $\begin{array}{l}\text { FAMILY } \\
\text { Scientific names }\end{array}$ & $\begin{array}{l}\text { Local } \\
\text { name }\end{array}$ & $\begin{array}{l}\text { Use } \\
\text { value } \\
\text { (UV) }\end{array}$ & $\begin{array}{l}\text { Part used and } \\
\text { application* }\end{array}$ & Preparation** & $\begin{array}{l}\text { Route of } \\
\text { administration }\end{array}$ & $\begin{array}{l}\text { Voucher } \\
\text { number }\end{array}$ \\
\hline $\begin{array}{l}\text { Maclura andamanica } \\
\text { (King ex Hook. f.) C. } \\
\text { C. Berg }\end{array}$ & $\begin{array}{l}\text { Chu- } \\
\text { Chae- } \\
\text { Bong }\end{array}$ & 0.02 & Fr: Flatulence & $\mathrm{Np}$ & Oral & $\begin{array}{l}\text { W. } \\
\text { Pongamornkul } \\
5622\end{array}$ \\
\hline \multicolumn{7}{|l|}{ MUSACEAE } \\
\hline $\begin{array}{l}\text { Musa acuminata } \\
\text { Colla }\end{array}$ & $\begin{array}{l}\text { Du-Ku- } \\
\text { Sae }\end{array}$ & 0.04 & Fl: Galactic & $\mathrm{De}$ & Oral & - \\
\hline \multicolumn{7}{|l|}{ MYRICACEAE } \\
\hline $\begin{array}{l}\text { Myrica esculenta } \\
\text { Buch. -Ham. ex D. } \\
\text { Don }\end{array}$ & $\begin{array}{l}\text { Bel-Sa- } \\
\text { Sae }\end{array}$ & 0.02 & $\begin{array}{l}\text { Fr, Bk: } \\
\text { Toothache }\end{array}$ & $\mathrm{Np}$ & Oral & $\begin{array}{l}\text { W. } \\
\text { Pongamornkul } \\
5343\end{array}$ \\
\hline \multicolumn{7}{|l|}{ MYRTACEAE } \\
\hline Psidium guajava L. & $\begin{array}{l}\text { Phu- } \\
\text { Sae- } \\
\text { Mo }\end{array}$ & 0.48 & $\begin{array}{l}\text { Lf :Diarrhea } \\
\text { Wp: Pain } \\
\text { Lf, Fr: Drunk, } \\
\text { Flatulence } \\
\text { Lf, Fr: Cough }\end{array}$ & $\begin{array}{l}\mathrm{Np} \\
\mathrm{De} \\
\mathrm{Np} \\
\mathrm{De}\end{array}$ & $\begin{array}{l}\text { Oral } \\
\text { Oral } \\
\text { Oral } \\
\text { Oral }\end{array}$ & $\begin{array}{l}\text { W. } \\
\text { Pongamornkul } \\
4983\end{array}$ \\
\hline $\begin{array}{l}\text { Syzygium cumini (L.) } \\
\text { Skeels }\end{array}$ & $\mathrm{Ka}-\mathrm{Mi}$ & 0.06 & $\begin{array}{l}\text { Bk: Diarrhea, } \\
\text { Blood tonic }\end{array}$ & $\mathrm{De}$ & Oral & $\begin{array}{l}\text { W. } \\
\text { Pongamornkul } \\
5016\end{array}$ \\
\hline \multicolumn{7}{|l|}{ PHYLLANTHACEAE } \\
\hline $\begin{array}{l}\text { Phyllanthus emblica } \\
\text { L. }\end{array}$ & $\begin{array}{l}\text { Ma-Lu- } \\
\text { Sae }\end{array}$ & 0.62 & $\begin{array}{l}\text { Fr, Ga: Cough } \\
\text { Lf: Fever } \\
\text { Rt: } \\
\text { Stomachache } \\
\text { Fr: Insomnia, } \\
\text { Sore throat } \\
\text { Fr: Tonic }\end{array}$ & $\begin{array}{l}\mathrm{Np} \\
\mathrm{De} \\
\mathrm{De} \\
\mathrm{Np} \\
\text { In }\end{array}$ & $\begin{array}{l}\text { Oral } \\
\text { Bath } \\
\text { Oral } \\
\text { Oral } \\
\text { Oral }\end{array}$ & $\begin{array}{l}\text { W. } \\
\text { Pongamornkul } \\
4551\end{array}$ \\
\hline \multicolumn{7}{|l|}{ PIPERACEAE } \\
\hline Piper betle L. & Pa-Lae & 0.02 & Lf: Shingles & Po & Poultices & - \\
\hline $\begin{array}{l}\text { Piper cf. wallichii } \\
\text { (Miq.) Hand.-Mazz. }\end{array}$ & $\begin{array}{l}\text { Pha- } \\
\text { Lae }\end{array}$ & 0.04 & $\begin{array}{l}\text { Rt: Backache } \\
\text { St: Laxative }\end{array}$ & $\begin{array}{l}\mathrm{De} \\
\mathrm{De}\end{array}$ & $\begin{array}{l}\text { Oral } \\
\text { Oral }\end{array}$ & $\begin{array}{l}\text { W. } \\
\text { Pongamornkul } \\
4529\end{array}$ \\
\hline \multicolumn{7}{|l|}{ PLANTAGINACEAE } \\
\hline Scoparia dulcis L. & $\begin{array}{l}\text { Ta-Si- } \\
\text { Ko }\end{array}$ & 0.14 & $\begin{array}{l}\text { Rt: Cough } \\
\text { Lf: Wounds } \\
\text { Wp: Fever, } \\
\text { Pollakiuria }\end{array}$ & $\begin{array}{l}\text { De } \\
\text { Po } \\
\text { De }\end{array}$ & $\begin{array}{l}\text { Oral } \\
\text { Poultices } \\
\text { Oral }\end{array}$ & $\begin{array}{l}\text { W. } \\
\text { Pongamornkul } \\
5034\end{array}$ \\
\hline
\end{tabular}

\footnotetext{
* Abbreviations for the plant parts: Bk = Bark; Bu = Bulbil; FI = Flower; Fr = Fruit; Ga = Gall; La = Latex; Lf = Leaf; Rh $=$ Rhizome; $\mathrm{Rt}=$ Root; $\mathrm{Sd}=$ Seed; $\mathrm{St}=\mathrm{Stem} ; \mathrm{Tu}=$ Tuber; $\mathrm{Wp}=$ Whole plant

**Abbreviations for the preparation methods: Bu = Burned; De = Decoction; In = Infusion; Np = Non-prepared ; Po $=$ Pounded
} 


\begin{tabular}{|c|c|c|c|c|c|c|}
\hline $\begin{array}{l}\text { FAMILY } \\
\text { Scientific names }\end{array}$ & $\begin{array}{l}\text { Local } \\
\text { name }\end{array}$ & $\begin{array}{l}\text { Use } \\
\text { value } \\
\text { (UV) }\end{array}$ & $\begin{array}{l}\text { Part used and } \\
\text { application* }\end{array}$ & Preparation** & $\begin{array}{l}\text { Route of } \\
\text { administration }\end{array}$ & $\begin{array}{l}\text { Voucher } \\
\text { number }\end{array}$ \\
\hline \multicolumn{7}{|l|}{ POACEAE } \\
\hline Coix lachryma-jobi L. & $\begin{array}{l}\text { Phong- } \\
\text { Mu-Thi }\end{array}$ & 0.08 & $\begin{array}{l}\text { Rt: Dizziness, } \\
\text { Hemorrhoids } \\
\text { Rt, Sd: } \\
\text { Nourishment }\end{array}$ & $\begin{array}{l}\mathrm{De} \\
\mathrm{De}\end{array}$ & $\begin{array}{l}\text { Oral } \\
\text { Oral }\end{array}$ & $\begin{array}{l}\text { W. } \\
\text { Pongamornkul } \\
1967\end{array}$ \\
\hline $\begin{array}{l}\text { Imperata cylindrica } \\
\text { (L.) Raeusch. }\end{array}$ & Khue & 0.06 & $\begin{array}{l}\text { Rt: Stones } \\
\text { Rt, Lf: } \\
\text { Diarrhea }\end{array}$ & $\begin{array}{l}\mathrm{De} \\
\mathrm{De}\end{array}$ & $\begin{array}{l}\text { Oral } \\
\text { Oral }\end{array}$ & $\begin{array}{l}\text { W. } \\
\text { Pongamornkul } \\
4956\end{array}$ \\
\hline \multicolumn{7}{|l|}{ POLYGALACEAE } \\
\hline Polygala chinensis L. & $\begin{array}{l}\text { Mai- } \\
\text { Mi- } \\
\text { Kang }\end{array}$ & 0.28 & $\begin{array}{l}\text { Rt: Flatulence, } \\
\text { Appetizing, } \\
\text { Muscle pain } \\
\text { Wp: } \\
\text { Stomachache }\end{array}$ & $\begin{array}{l}\mathrm{De} \\
\mathrm{De}\end{array}$ & $\begin{array}{l}\text { Oral } \\
\text { Oral }\end{array}$ & $\begin{array}{l}\text { W. } \\
\text { Pongamornkul } \\
5248\end{array}$ \\
\hline \multicolumn{7}{|l|}{ PRIMULACEAE } \\
\hline $\begin{array}{l}\text { Embelia sessiliflora } \\
\text { Kurz }\end{array}$ & $\begin{array}{l}\text { Blae- } \\
\text { Blang }\end{array}$ & 0.02 & $\begin{array}{l}\text { Fr: } \\
\text { Anthelmintic }\end{array}$ & Po & Oral & $\begin{array}{l}\text { W. } \\
\text { Pongamornkul } \\
4476\end{array}$ \\
\hline $\begin{array}{l}\text { Maesa glomerata K. } \\
\text { Larsen \& C. M. Hu }\end{array}$ & $\begin{array}{l}\text { Choi- } \\
\text { Ne-Du }\end{array}$ & 0.08 & $\begin{array}{l}\text { Lf: Wound } \\
\text { and boils }\end{array}$ & Po & Poultices & \\
\hline \multicolumn{7}{|l|}{ PTERIDACEAE } \\
\hline $\begin{array}{l}\text { Onychium } \\
\text { siliculosum (Desv.) C. } \\
\text { Chr. }\end{array}$ & $\begin{array}{l}\text { Pho- } \\
\text { Khai- } \\
\text { Mi }\end{array}$ & 0.06 & $\begin{array}{l}\text { Rt: } \\
\text { Stomachache, } \\
\text { Rt: Lumbago } \\
\text { Rt: } \\
\text { Postpartum } \\
\text { period }\end{array}$ & $\begin{array}{l}\text { Po } \\
\text { In } \\
\text { De }\end{array}$ & $\begin{array}{l}\text { Oral } \\
\text { Oral } \\
\text { Oral }\end{array}$ & $\begin{array}{l}\text { W. } \\
\text { Pongamornkul } \\
5251\end{array}$ \\
\hline \multicolumn{7}{|l|}{ RHAMNACEAE } \\
\hline $\begin{array}{l}\text { Berchemia floribunda } \\
\text { (Wall.) Brongn. }\end{array}$ & $\begin{array}{l}\text { Ta-Si- } \\
\text { Pe-Ma- } \\
\text { Ho }\end{array}$ & 0.16 & $\begin{array}{l}\text { Rt: Stones } \\
\text { Bk: } \\
\text { Hemorrhoids } \\
\text { St: } \\
\text { Nourishment } \\
\text { St, Lf: Pain } \\
\text { Rt, Bk: } \\
\text { Paralysis }\end{array}$ & $\begin{array}{l}\text { De } \\
\text { De } \\
\text { De } \\
\text { De } \\
\text { De }\end{array}$ & $\begin{array}{l}\text { Oral } \\
\text { Oral } \\
\text { Oral } \\
\text { Oral } \\
\text { Oral }\end{array}$ & $\begin{array}{l}\text { W. } \\
\text { Pongamornkul } \\
5585\end{array}$ \\
\hline
\end{tabular}

\footnotetext{
* Abbreviations for the plant parts: Bk = Bark; Bu = Bulbil; FI = Flower; Fr = Fruit; Ga = Gall; La = Latex; Lf = Leaf; Rh $=$ Rhizome; Rt = Root; Sd = Seed; St = Stem; Tu = Tuber; $\mathrm{Wp}=$ Whole plant

**Abbreviations for the preparation methods: Bu = Burned; De = Decoction; In = Infusion; Np = Non-prepared ; Po $=$ Pounded
} 


\begin{tabular}{|c|c|c|c|c|c|c|}
\hline $\begin{array}{l}\text { FAMILY } \\
\text { Scientific names }\end{array}$ & $\begin{array}{l}\text { Local } \\
\text { name }\end{array}$ & $\begin{array}{l}\text { Use } \\
\text { value } \\
\text { (UV) }\end{array}$ & $\begin{array}{l}\text { Part used and } \\
\text { application* }\end{array}$ & Preparation** & $\begin{array}{l}\text { Route of } \\
\text { administration }\end{array}$ & $\begin{array}{l}\text { Voucher } \\
\text { number }\end{array}$ \\
\hline $\begin{array}{l}\text { Prunus campanulata } \\
\text { Maxim. }\end{array}$ & $\begin{array}{l}\text { Khong- } \\
\text { Kho }\end{array}$ & 0.04 & $\begin{array}{l}\text { Bk: Muscle } \\
\text { pain, } \\
\text { Nourishment }\end{array}$ & $\mathrm{De}$ & Oral & $\begin{array}{l}\text { W. } \\
\text { Pongamornkul } \\
4579\end{array}$ \\
\hline Rubus ellipticus Sm. & $\begin{array}{l}\text { Wae- } \\
\text { Chu- } \\
\text { Sae }\end{array}$ & 0.02 & Lf: Canker & $\mathrm{Bu}$ & Poultices & $\begin{array}{l}\text { W. } \\
\text { Pongamornkul } \\
4925\end{array}$ \\
\hline \multicolumn{7}{|l|}{ RUBIACEAE } \\
\hline $\begin{array}{l}\text { Morinda angustifolia } \\
\text { Roxb. }\end{array}$ & Khu & 0.02 & $\begin{array}{l}\text { Rt, St: } \\
\text { Jaundice }\end{array}$ & $\mathrm{De}$ & Oral & $\begin{array}{l}\text { W. } \\
\text { Pongamornkul } \\
4849\end{array}$ \\
\hline Mussaenda sp. & $\begin{array}{l}\text { Mae- } \\
\text { Cha- } \\
\text { Kwa }\end{array}$ & 0.04 & $\begin{array}{l}\text { Rt, St: Pain } \\
\text { Lf: Itching }\end{array}$ & $\begin{array}{l}\mathrm{De} \\
\mathrm{De}\end{array}$ & $\begin{array}{l}\text { Oral } \\
\text { Oral }\end{array}$ & $\begin{array}{l}\text { W. } \\
\text { Pongamornkul } \\
4581\end{array}$ \\
\hline $\begin{array}{l}\text { Paederia pilifera } \\
\text { Hook. f. }\end{array}$ & $\begin{array}{l}\text { Thui-I- } \\
\text { Mi }\end{array}$ & 0.04 & $\begin{array}{l}\text { Lf: Treating } \\
\text { flatulence } \\
\text { Wp: } \\
\text { Constipation }\end{array}$ & $\begin{array}{l}\mathrm{Np} \\
\mathrm{De}\end{array}$ & $\begin{array}{l}\text { Oral } \\
\text { Oral }\end{array}$ & $\begin{array}{l}\text { W. } \\
\text { Pongamornkul } \\
\text { et al. } 5091\end{array}$ \\
\hline $\begin{array}{l}\text { Uncaria cordata } \\
\text { (Lour.) Merr. }\end{array}$ & $\begin{array}{l}\text { Ta-Si- } \\
\text { Khi }\end{array}$ & 0.06 & $\begin{array}{l}\text { Rt: Muscle } \\
\text { pain } \\
\text { Wp: Leg pain }\end{array}$ & $\begin{array}{l}\mathrm{De} \\
\mathrm{De}\end{array}$ & $\begin{array}{l}\text { Oral } \\
\text { Oral }\end{array}$ & $\begin{array}{l}\text { W. } \\
\text { Pongamornkul } \\
5596\end{array}$ \\
\hline \multicolumn{7}{|l|}{ RUTACEAE } \\
\hline $\begin{array}{l}\text { Clausena excavata } \\
\text { Burm. f. }\end{array}$ & $\begin{array}{l}\text { Se-Na- } \\
0\end{array}$ & 0.04 & $\begin{array}{l}\text { Lf, Wp: } \\
\text { Intoxication, } \\
\text { Muscle pain }\end{array}$ & $\mathrm{De}$ & Bath & $\begin{array}{l}\text { W. } \\
\text { Pongamornkul } \\
4598\end{array}$ \\
\hline $\begin{array}{l}\text { Toddalia asiatica (L.) } \\
\text { Lam. }\end{array}$ & $\begin{array}{l}\text { Lae- } \\
\text { Kho-Mi }\end{array}$ & 0.02 & $\begin{array}{l}\text { Rt: Muscle } \\
\text { pain }\end{array}$ & $\mathrm{De}$ & Oral & $\begin{array}{l}\text { W. } \\
\text { Pongamornkul } \\
5142\end{array}$ \\
\hline Zanthoxylum sp. & $\begin{array}{l}\text { Mang- } \\
\text { Khay }\end{array}$ & 0.14 & $\begin{array}{l}\text { Bk, Fr: } \\
\text { Toothache }\end{array}$ & $\mathrm{Np}$ & Oral & $\begin{array}{l}\text { W. } \\
\text { Pongamornkul } \\
5168\end{array}$ \\
\hline \multicolumn{7}{|l|}{ SALICACEAE } \\
\hline $\begin{array}{l}\text { Flacourtia jangomas } \\
\text { (Lour.) Raeusch. }\end{array}$ & $\begin{array}{l}\text { Phli- } \\
\text { Sae }\end{array}$ & 0.06 & $\begin{array}{l}\text { Rt: } \\
\text { Stomachache } \\
\text { Fr: Cough }\end{array}$ & $\begin{array}{l}\mathrm{De} \\
\mathrm{Np}\end{array}$ & $\begin{array}{l}\text { Oral } \\
\text { Oral }\end{array}$ & $\begin{array}{l}\text { W. } \\
\text { Pongamornkul } \\
\text { et al. } 5118\end{array}$ \\
\hline
\end{tabular}

\footnotetext{
* Abbreviations for the plant parts: Bk = Bark; Bu = Bulbil; FI = Flower; Fr = Fruit; Ga = Gall; La = Latex; Lf = Leaf; Rh $=$ Rhizome; Rt = Root; Sd = Seed; St = Stem; Tu = Tuber; $\mathrm{Wp}=$ Whole plant

**Abbreviations for the preparation methods: $\mathrm{Bu}=$ Burned; $\mathrm{De}=$ Decoction; $\mathrm{In}=$ Infusion; $\mathrm{Np}=$ Non-prepared ; Po $=$ Pounded
} 


\begin{tabular}{|c|c|c|c|c|c|c|}
\hline $\begin{array}{l}\text { FAMILY } \\
\text { Scientific names }\end{array}$ & $\begin{array}{l}\text { Local } \\
\text { name }\end{array}$ & $\begin{array}{l}\text { Use } \\
\text { value } \\
\text { (UV) }\end{array}$ & $\begin{array}{l}\text { Part used and } \\
\text { application* }\end{array}$ & Preparation** & $\begin{array}{l}\text { Route of } \\
\text { administration }\end{array}$ & $\begin{array}{l}\text { Voucher } \\
\text { number }\end{array}$ \\
\hline $\begin{array}{l}\text { Osyris lanceolata } \\
\text { Hochst. \& Steud. }\end{array}$ & $\begin{array}{l}\text { Ta-Si- } \\
\text { Ko }\end{array}$ & 0.28 & $\begin{array}{l}\text { Rt: Muscle } \\
\text { pain, } \\
\text { Hemorrhoids, } \\
\text { Cough, } \\
\text { Appetizing, } \\
\text { Diarrhea, } \\
\text { Dysuria }\end{array}$ & De & Oral & $\begin{array}{l}\text { W. } \\
\text { Pongamornkul } \\
4540\end{array}$ \\
\hline \multicolumn{7}{|l|}{ SAPOTACEAE } \\
\hline $\begin{array}{l}\text { Xantolis burmanica } \\
\text { (Collett \& Hemsl.) P. } \\
\text { Royen }\end{array}$ & $\begin{array}{l}\text { Sai- } \\
\text { Nu-Thi }\end{array}$ & 0.06 & $\begin{array}{l}\text { Bk: } \\
\text { Stomachache, } \\
\text { Malnutrition }\end{array}$ & $\mathrm{De}$ & Oral & $\begin{array}{l}\text { W. } \\
\text { Pongamornkul } \\
\text { \& Pongsri } \\
3165\end{array}$ \\
\hline \multicolumn{7}{|l|}{ SAURURACEAE } \\
\hline $\begin{array}{l}\text { Houttuynia cordata } \\
\text { Thunb. }\end{array}$ & $\begin{array}{l}\text { Ta-Na- } \\
\text { Khi-Du }\end{array}$ & 0.04 & $\begin{array}{l}\text { Lf: Dizziness } \\
\text { Lf: Swellings }\end{array}$ & $\begin{array}{l}\text { Po } \\
\text { Po }\end{array}$ & $\begin{array}{l}\text { Smell } \\
\text { Poultices }\end{array}$ & $\begin{array}{l}\text { W. } \\
\text { Pongamornkul } \\
3075\end{array}$ \\
\hline \multicolumn{7}{|l|}{ SCROPHULARIACEAE } \\
\hline $\begin{array}{l}\text { Buddleja asiatica } \\
\text { Lour. }\end{array}$ & $\begin{array}{l}\text { Phu- } \\
\text { Chi- } \\
\text { Buy }\end{array}$ & 0.14 & $\begin{array}{l}\text { St, Lf: } \\
\text { Postpartum } \\
\text { period } \\
\text { Lf: Wound } \\
\text { and Boils } \\
\text { Wp: Drunk } \\
\text { Rt, St and Lf: } \\
\text { Stones }\end{array}$ & $\begin{array}{l}\text { De } \\
\text { Po } \\
\text { De } \\
\text { De }\end{array}$ & $\begin{array}{l}\text { Oral } \\
\text { Liniment } \\
\text { Oral } \\
\text { Oral }\end{array}$ & $\begin{array}{l}\text { W. } \\
\text { Pongamornkul } \\
4544\end{array}$ \\
\hline \multicolumn{7}{|l|}{ SIMAROUBACEAE } \\
\hline $\begin{array}{l}\text { Picrasma javanica } \\
\text { Blume }\end{array}$ & $\begin{array}{l}\text { Ya-Pa- } \\
\text { Chu }\end{array}$ & 0.24 & $\begin{array}{l}\text { Bk: Muscle } \\
\text { pain, Tonic, } \\
\text { Diabetes } \\
\text { Bk: } \\
\text { Haemorrhagic } \\
\text { Fever } \\
\text { St: } \\
\text { Hypertension }\end{array}$ & $\begin{array}{l}\text { De } \\
\text { In } \\
\text { De }\end{array}$ & $\begin{array}{l}\text { Oral } \\
\text { Oral } \\
\text { Oral }\end{array}$ & $\begin{array}{l}\text { W. } \\
\text { Pongamornkul } \\
5684\end{array}$ \\
\hline \multicolumn{7}{|l|}{ SOLANACEAE } \\
\hline Physalis angulata L. & $\begin{array}{l}\text { Thu- } \\
\text { Chi- } \\
\text { Bong }\end{array}$ & 0.06 & $\begin{array}{l}\text { Rt: Flatulence } \\
\text { St: } \\
\text { Nephropathy }\end{array}$ & $\begin{array}{l}\mathrm{De} \\
\mathrm{De}\end{array}$ & $\begin{array}{l}\text { Oral } \\
\text { Oral }\end{array}$ & $\begin{array}{l}\text { W. } \\
\text { Pongamornkul } \\
3525\end{array}$ \\
\hline
\end{tabular}

\footnotetext{
* Abbreviations for the plant parts: Bk = Bark; Bu = Bulbil; FI = Flower; Fr = Fruit; Ga = Gall; La = Latex; Lf = Leaf; Rh

$=$ Rhizome; Rt = Root; Sd = Seed; St = Stem; Tu = Tuber; $\mathrm{Wp}=$ Whole plant

**Abbreviations for the preparation methods: $\mathrm{Bu}=$ Burned; $\mathrm{De}=$ Decoction; $\mathrm{In}=$ Infusion; $\mathrm{Np}=$ Non-prepared ; Po $=$ Pounded
} 


\begin{tabular}{|c|c|c|c|c|c|c|}
\hline $\begin{array}{l}\text { FAMILY } \\
\text { Scientific names }\end{array}$ & $\begin{array}{l}\text { Local } \\
\text { name }\end{array}$ & $\begin{array}{l}\text { Use } \\
\text { value } \\
\text { (UV) }\end{array}$ & $\begin{array}{l}\text { Part used and } \\
\text { application* }\end{array}$ & Preparation** & $\begin{array}{l}\text { Route of } \\
\text { administration }\end{array}$ & $\begin{array}{l}\text { Voucher } \\
\text { number }\end{array}$ \\
\hline $\begin{array}{l}\text { Solanum anguivi } \\
\text { Lam. }\end{array}$ & $\begin{array}{l}\text { Khang- } \\
\text { Khae }\end{array}$ & 0.16 & $\begin{array}{l}\text { St, Fr: Cough } \\
\text { Fr: Pain, Sore } \\
\text { throat } \\
\text { Fr: Muscle } \\
\text { pain }\end{array}$ & $\begin{array}{l}\mathrm{Np}, \mathrm{De} \\
\mathrm{Np} \\
\mathrm{De}\end{array}$ & $\begin{array}{l}\text { Oral } \\
\text { Oral } \\
\text { Oral }\end{array}$ & $\begin{array}{l}\text { W. } \\
\text { Pongamornkul } \\
4550\end{array}$ \\
\hline Solanum torvum Sw. & $\begin{array}{l}\text { Ta- } \\
\text { Khang- } \\
\text { Khae }\end{array}$ & 0.02 & $\begin{array}{l}\text { Fr: Muscle } \\
\text { pain }\end{array}$ & $\mathrm{De}$ & Oral & $\begin{array}{l}\text { W. } \\
\text { Pongamornkul } \\
04208\end{array}$ \\
\hline \multicolumn{7}{|l|}{ VITACEAE } \\
\hline Cissus javana DC. & $\begin{array}{l}\text { Ta-Si- } \\
\text { Si-Mo- } \\
\text { Pang }\end{array}$ & 0.02 & Lf: Itching & $\mathrm{Bu}$ & Poultices & $\begin{array}{l}\text { W. } \\
\text { Pongamornkul } \\
4987\end{array}$ \\
\hline $\begin{array}{l}\text { Tetrastigma } \\
\text { obovatum Gagnep. }\end{array}$ & $\begin{array}{l}\text { Kuey- } \\
\text { Cha- } \\
\text { Sae }\end{array}$ & 0.02 & $\begin{array}{l}\text { Fr: Morning } \\
\text { sickness }\end{array}$ & $\mathrm{Np}$ & Oral & $\begin{array}{l}\text { W. } \\
\text { Pongamornkul } \\
4457\end{array}$ \\
\hline \multicolumn{7}{|l|}{ ZINGIBERACEAE } \\
\hline $\begin{array}{l}\text { Boesenbergia } \\
\text { rotunda (L.) Mansf. }\end{array}$ & $\begin{array}{l}\text { Pho- } \\
\text { Sa-Du }\end{array}$ & 0.06 & Rh: Flatulence & $\mathrm{Np}$ & Oral & $\begin{array}{l}\text { W. } \\
\text { Pongamornkul } \\
3221\end{array}$ \\
\hline Curcuma longa L. & $\begin{array}{l}\text { Yae- } \\
\text { Bang }\end{array}$ & 0.9 & $\begin{array}{l}\text { Rh: } \\
\text { Stomachache } \\
\text { Rh: Wounds } \\
\text { Rh: } \\
\text { Flatulence, } \\
\text { Gastritis } \\
\text { Rh: Insect } \\
\text { Bites, Itching } \\
\text { Rh, Fl: Fever, } \\
\text { Cough }\end{array}$ & $\begin{array}{l}\mathrm{Np} \\
\mathrm{Po} \\
\mathrm{De} \\
\mathrm{Po} \\
\mathrm{De}\end{array}$ & $\begin{array}{l}\text { Oral } \\
\text { Poultices } \\
\text { Oral } \\
\text { Liniment } \\
\text { Oral }\end{array}$ & $\begin{array}{l}\text { W. } \\
\text { Pongamornkul } \\
2004\end{array}$ \\
\hline $\begin{array}{l}\text { Curcuma rubescens } \\
\text { Roxb. }\end{array}$ & $\begin{array}{l}\text { Pho- } \\
\text { Khae }\end{array}$ & 0.12 & $\begin{array}{l}\text { Rh: Treating } \\
\text { flatulence } \\
\text { Rh: Fever } \\
\text { Rh: } \\
\text { Stomachache }\end{array}$ & $\begin{array}{l}\mathrm{De} \\
\mathrm{De} \\
\mathrm{Np}\end{array}$ & $\begin{array}{l}\text { Oral } \\
\text { Bath } \\
\text { Oral }\end{array}$ & - \\
\hline $\begin{array}{l}\text { Hedychium } \\
\text { stenopetalum Lodd. }\end{array}$ & $\begin{array}{l}\text { Pho- } \\
\text { Sai }\end{array}$ & 0.02 & $\begin{array}{l}\text { Rh: } \\
\text { Stomachache }\end{array}$ & $\mathrm{Np}$ & Oral & $\begin{array}{l}\text { W. } \\
\text { Pongamornkul } \\
\text { et al. } 5103\end{array}$ \\
\hline Zingiber kerrii Craib & $\begin{array}{l}\text { Pho- } \\
\text { Ang }\end{array}$ & 0.12 & $\begin{array}{l}\text { Rh, Fl: } \\
\text { Flatulence } \\
\text { Rh: } \\
\text { Stomachache } \\
\text { Fl: } \\
\text { Nourishment }\end{array}$ & $\begin{array}{l}\mathrm{De} \\
\mathrm{Np} \\
\mathrm{Np}\end{array}$ & $\begin{array}{l}\text { Oral } \\
\text { Oral } \\
\text { Oral }\end{array}$ & - \\
\hline \multicolumn{7}{|c|}{$\begin{array}{l}\text { * Abbreviations for the plant parts: Bk = Bark; Bu = Bulbil; FI = Flower; Fr = Fruit; Ga = Gall; La = Latex; Lf = Leaf; Rh } \\
\text { = Rhizome; Rt = Root; Sd = Seed; St = Stem; Tu = Tuber; Wp = Whole plant }\end{array}$} \\
\hline
\end{tabular}




\begin{tabular}{|c|c|c|c|c|c|c|}
\hline $\begin{array}{l}\text { FAMILY } \\
\text { Scientific names }\end{array}$ & $\begin{array}{l}\text { Local } \\
\text { name }\end{array}$ & $\begin{array}{l}\text { Use } \\
\text { value } \\
\text { (UV) }\end{array}$ & $\begin{array}{l}\text { Part used and } \\
\text { application* }\end{array}$ & Preparation** & $\begin{array}{l}\text { Route of } \\
\text { administration }\end{array}$ & $\begin{array}{l}\text { Voucher } \\
\text { number }\end{array}$ \\
\hline $\begin{array}{l}\text { Zingiber purpureum } \\
\text { Roscoe }\end{array}$ & Pa-Lui & 0.68 & $\begin{array}{l}\text { Rh: } \\
\text { Postpartum } \\
\text { period } \\
\text { Rh: } \\
\text { Flatulence, } \\
\text { Hiccup, } \\
\text { Hypertension, } \\
\text { Stomachache, } \\
\text { Diarrhea } \\
\text { Rh: Sprain }\end{array}$ & $\begin{array}{l}\text { De } \\
\text { De } \\
\text { Po }\end{array}$ & $\begin{array}{l}\text { Oral } \\
\text { Oral } \\
\text { Poultices }\end{array}$ & $\begin{array}{l}\text { W. } \\
\text { Pongamornkul } \\
4286\end{array}$ \\
\hline $\begin{array}{l}\text { Zingiber officinale } \\
\text { Roscoe }\end{array}$ & Eng & 0.22 & $\begin{array}{l}\text { Rh: Cough } \\
\text { Rh: Flatulence } \\
\text { Rh: } \\
\text { Stomachache } \\
\text { Rh: Fever }\end{array}$ & $\begin{array}{l}\mathrm{Np} \\
\mathrm{Bu} \\
\mathrm{De} \\
\mathrm{De}\end{array}$ & $\begin{array}{l}\text { Oral } \\
\text { Oral } \\
\text { Oral } \\
\text { Bath }\end{array}$ & - \\
\hline $\begin{array}{l}\text { Zingiber ottensii } \\
\text { Valeton }\end{array}$ & $\begin{array}{l}\text { Pa-Lui- } \\
\text { Sa }\end{array}$ & 0.44 & $\begin{array}{l}\text { Rh: Flatulence } \\
\text { Rh: } \\
\text { Stomachache, } \\
\text { Dizziness, } \\
\text { Diarrhea }\end{array}$ & $\begin{array}{l}\mathrm{Np}, \mathrm{Bu} \\
\mathrm{De}\end{array}$ & $\begin{array}{l}\text { Oral } \\
\text { Oral }\end{array}$ & $\begin{array}{l}\text { W. } \\
\text { Pongamornkul } \\
2001\end{array}$ \\
\hline \multicolumn{7}{|c|}{$\begin{array}{l}\text { * Abbreviations for the plant parts: Bk = Bark; Bu = Bulbil; Fl = Flower; } \mathrm{Fr}=\text { Fruit; Ga = Gall; La = Latex; Lf = Leaf; Rh } \\
\text { = Rhizome; Rt = Root; Sd = Seed; St = Stem; Tu = Tuber; Wp = Whole plant }\end{array}$} \\
\hline
\end{tabular}

Use recorded and use categories

There was a total of 930 use reports which were categorized into 22 use categories. The digestive system category was the largest group with $39 \mathrm{spp}$. and 143 reports (Table 3). Other use categories that had more than 100 use records included muscular-skeletal system, respiratory system, and the pain and injuries categories, respectively. 
Table 3

The number of used reports, species and IAR of each use category which were reported from 50 Pwo informants, from 8 villages in Na Kian sub-district, Omkoi district, Chiang Mai provinces, Thailand.

\begin{tabular}{|c|c|c|c|}
\hline Use categories & No. of used report $\left(N_{u r}\right)$ & No. of species used $\left(N_{t}\right)$ & IAR \\
\hline Injuries & 126 & 15 & 0.89 \\
\hline Respiratory system & 138 & 35 & 0.75 \\
\hline Digestive system & 143 & 39 & 0.73 \\
\hline Muscular-skeletal system & 140 & 43 & 0.70 \\
\hline Pain & 133 & 50 & 0.63 \\
\hline Endocrine system & 14 & 6 & 0.62 \\
\hline Nutritional disorders & 46 & 20 & 0.58 \\
\hline poisonings & 15 & 7 & 0.57 \\
\hline Infections & 67 & 32 & 0.53 \\
\hline Neoplasms & 5 & 3 & 0.50 \\
\hline Skin/Subcutaneous Cellular Tissue & 14 & 8 & 0.46 \\
\hline Pregnancy/Birth/Puerperium & 17 & 10 & 0.44 \\
\hline Other & 19 & 12 & 0.39 \\
\hline Genitourinary system & 28 & 19 & 0.33 \\
\hline Circulatory system & 8 & 7 & 0.14 \\
\hline Blood system & 2 & 2 & 0 \\
\hline Metabolic system & 2 & 2 & 0 \\
\hline Inflammation & 2 & 2 & 0 \\
\hline Sensory system & 2 & 2 & 0 \\
\hline III-defined symptoms & 6 & 6 & 0 \\
\hline Mental disorders & 4 & 4 & 0 \\
\hline Abnormalities & 1 & 1 & - \\
\hline
\end{tabular}

Plant part uses, life forms, and method of preparation

Leaves were the most preferred part for use, followed by rhizomes/roots, and stems (Fig. 3). These parts contributed to more than $50 \%$ of total use-reports. Herbs were the most preferred life form, followed by trees. These life forms contributed to more than $50 \%$ of total species found in this study (Fig. 4). Decoction was the main method of preparation (Fig. 5) with half of use-reports prepared by this method.

\section{Informant Agreement Ration (IAR)}


The highest IAR (= 0.89) was observed in the injuries use category (Table 3 ). This category had 126 use-reports and 15 species used. The IAR of the digestive system category, which had highest number of use-reports, was 0.73 which was the third followed by the respiratory system category. Many use categories had IAR $=0$ because the number of use-reports was equal to the number of species used. The IAR of the abnormalities category could not be calculated because the number of use-reports was 1 which made the divider in IAR formula equal to 0.

\section{Plant Use Values (UV)}

Use values ranged from 0.02 (use-report $=1$ ) to 0.94 (use-report $=47$ ) (Table 2). The most used species was Ageratina adenophora (Spreng.) R.M.King \& H.Rob. (Asteraceae) which was used to stop bleeding. There were other three species in Asteraceae that also had high use values, including Blumea balsamifera (L) DC. (UV = 0.92), Chromolaena odorata (L.) R.M.King \& H.Rob. (UV = 0.88) and Elephantopus scaber L. (UV = 0.72). Chromolaena odorata (L.) R.M.King \& H.Rob. had the same uses as A. adenophora (Spreng.) R.M.King \& H. Rob. while the other two were used differently. Example of other species with high UV included Curcuma longa L., Garcinia pedunculata Roxb. ex Buch.-Ham., Acorus calamus L., Zingiber purpureum Roscoe., Phyllanthus emblica L. and Betula alnoides Buch.-Ham. ex G.Don.

Comparing ethnomedicinal plants used by Pwo People to medicinal plants in Thailand

There were 10 species that were not presented in Phumthum et al. [19] which reviewed all medicinal plants reported in ethnobotanical studies across Thailand. These species included: Phoenix loureiroi Kunth, Gerbera piloselloides (L.) Cass., Garcinia pedunculata Roxb. ex Buch.-Ham., Tupistra muricata (Gagnep.) N.Tanaka, Maclura andamanica (King ex J.D. Hooker) C.C. Berg, Myrica esculenta Buch.-Ham. ex D. Don, Prunus campanulata Maxim., Osyris lanceolata Hochst. \& Steud., Solanum anguivi Lam., and Curcuma rubescens Roxb. According to the best of our knowledge, these plants, except Prunus campanulata Maxim., was firstly published for their medicinal properties in Thailand.

\section{Discussion}

\section{Diversity of ethnomedicinal plants used by Pwo People}

The number of ethnomedicinal plants recorded from the Pwo in this study was as high for other ethnicities in northern Thailand [8,20-21] but lower than in the study of Tangjitman et al. (2013) [11], which studied Sgaw Karen living in wide geographical ranges. This indicated that the Pwo also possess great knowledge of ethnomedicinal plants. It should be noted that the Pwo people are distributed in many parts of northern Thailand. The adaptation to the new land would result in the use of new plants [19]. More ethnobotanical investigation of the Pwo people in other locations could result in a great compilation of ethnobotanical knowledge.

The dominant families in this study were in accordance with those in other ethnobotanical studies in northern Thailand [8,11,20-22]. These families, Asteraceae, Fabaceae, and Zingiberaceae, are cosmopolitan families commonly known for their medicinal properties [23]. Moreover, these families were also listed as some of the largest families in Thailand [24].

Use of ethnomedicinal plants: parts; life forms; and preparation 
Leaves are gathered more easily than other plant parts [25]. and are also full of secondary metabolites [26]. Moreover, gathering leaves is more sustainable than other parts because it rarely affects the survival or the reproduction of the plants. Leaves are one of the plant organs which contain many active compounds some of which possess valuable medicinal properties for humans [23]. Hypogenous organs, roots and rhizome, were also preferred by Pwo, the same as other ethnic groups [4,22,27]. These hypogenous organs normally possess a high content of secondary metabolites [28]. which could be used to cure many ailments.

Among the Pwo, herbs were the most frequently used life form, in accordance with ethnomedicinal studies for many other ethnic groups in northern Thailand [20-22]. This life form was easy to harvest and the preparation methods and extraction of active ingredient also was also easier than for other life forms.

Most ethnomedicinal plant parts were prepared by boiling in hot water (decoction). This is one of the easiest and simplest methods to extracted active ingredients from medicinal plants [29]. This method is commonly practiced among the ethnic groups in northern Thailand [30-31]. and other parts of the world [32-33].

\section{Ethnobotanical indices}

Use values (UV) indicate the relative importance of a specific species used by the informants. Plants have high UV when they are extensively used and known by the informants. Many plants that were reported with high UV in this study were common species: most were widespread or commonly cultivated according to a study in the nearby area [34]. Some of them were widespread exotic species: Ageratina adenophora (Spreng.) R.M. King \& H. Rob. and Chromolaena odorata (L.) R.M. King \& H.Rob. This indicated the adaptation of the indigenous people to their new environments [20]. Most of these plants were used to cure common acute symptoms like bleeding wounds, diarrhea, or common illnesses like cough and headache. So it could be said that one of the factors that promoted the uses of plants in Pwo people is their common appearance in the area. This would allow the local people to become familiar with their properties, resulting in high probability of including them as part of the culture [35]. However, it should be noted that there was a species which had high UV but low prevalence and many species which had very low UVs but were also common species in the areas, e.g. Senegalia rugata (Lam.) Britton \& Rose; Toddalia asiatica (L.) Lam.; Solanum torvum Sw., etc. These species were commonly found and used as vegetable in the studied site. It could be said that low UV of these plants did not indicate that they were unimportant but indicated that the local people were unaware of their medicinal properties [23]. That meant the medicinal knowledge of these plants was at risk of disappearing from the community [36].

One of the notable species is Garcinia pedunculata Roxb. ex Buch.-Ham (called 'Ko-kuy-sa' by local people). The fruit of plant used to treat many ailments, for example diabetes, flatulence, lumbago, etc. (Table 1). However, only a single tree was found in the nearby forest of the studied area but its fruits were widely and popularly used by Pwo people. The extensive use of this plant seemed to be limited to the Pwo people.

There were five use categories which reported both high informant agreement ratios (IAR) and high numbers of usereports. This indicated that a well-defined selection of species is culturally important for treating these illnesses [17]. Normally the IAR also related to the prevalence of these ailments in the studied area. Most of the informants in this study were farmers so many ailments which related to the hard works in their fields showed high IARs. These included the ailments in the injuries, muscular-skeletal system, and pain categories. The respiratory systems category also had high IAR and Nur. Plants are commonly used to cure respiratory disorders in many parts of the world [37]. Most of these plants in this category were used to treat the common cold and other related symptoms

Page 24/32 
like cough which were common disorders in children [38]. Another use category with both high IAR and Nur among Pwo people was digestive system disorders. Digestive diseases are always reported as among the most important disorders among the ethnic groups in Thailand [4,20-22] The cause of the ailments could be drinking water from forest streams without boiling [4]. It should be noted that high IAR can also help in discovering potentially effective medicinal plants [17].

\section{Comparison of ethnomedicinal plants}

Among the 122 species found in this study there were 10 species (8.2\%) whose uses were reported in Thailand for the first time. Although the number was not large, but it should be noted that more than 2,000 species were reported in Phumthum et al. (2018) [19] and the accumulation curve indicated that it represents a nearly complete set of used medicinal species in Thailand [19]. This shown that there is still a gap in our knowledge of plant used by local people. Especially, from the ethnic groups which were never been ethnobotanical studied before. Even though they lived in the same area other groups which have been studied for their ethnobotany before. It should be noted that only the plant names, but not use-reports, were compared.

Additionally, there was a significant relation between the UV of plants used by Pwo people and those reported in Phumthum et al. (2018) [19] ( $r=0.53, p<0.01$, Pearson's correlation test). The reason behind this could be that most of ethnomedicinal species with high UV were common species which could be found throughout Thailand, so users have more chance to encounter the same common plants which normally tended to have high UVs according to their frequency of appearance [39].

\section{Conclusion}

Ethnomedicinal plants still play important roles in the daily life of the Pwo people as witnessed by the great varieties of plants used. Moreover, their knowledge was differed from the Sgaw Karen who lived in the same area. The divergence of ethnomedicinal knowledge among the ethnic groups demonstrated the importance of ethnobotanical studies. In northern Thailand there are many ethnic groups on which few or no ethnobotanical studies have been carried out, so it is urgent at least to document their knowledge before erosion as the result of urbanization or modernization. The loss of this knowledge means the loss of opportunities for discovering and developing many new products from them.

\section{Declarations}

Competing interests

The authors declare that they have no competing interests.

Funding

This research was partly supported by Research Center in Bioresources for Agriculture, Industry and Medicine, Chiang Mai University.

Authors' contributions

WP and NM carried out the field research. Al supervised the work. PP, BP and Al analyzed the data and wrote the manuscript. All authors read and approved the final manuscript.

Acknowledgements

We thank Mae Jang Watersheds Management Unit, Ministry of Natural Resources and Environment, Na kian Subdistrict Administrative Organization (SAO) and Na Kian Agricultural Development Projects Station for 
accommodation and helps in fieldwork. We also appreciate all villagers who provided us a warm welcome and the willing to inform us about their ethnomedicinal knowledge. I am very grateful to the Center of Excellence in Bioresources for Agriculture, Industry and Medicine, Chiang Mai University for partly providing financial support. Availability of data and materials

Data generated and analyzed in this study are included in this published article.

Ethics approval and consent to participate

The authors informed the head of village about purposed of the studied and asked for permission from individuals' informants.

\section{References}

1. Vijendra N, Kumar KP : Traditional knowledge on ethno-medicinal uses prevailing in tribal pockets of Chhindwara and Betul Districts, Madhya Pradesh, India. African. Journal of Pharmacy and Pharmacology 2010; 4: 662-670.

2. United Nations : World Urbanization Prospects; The 2014 Revision United Nations: New York; 2014.

3. WHO: Traditional Medicine-Growing Needs and Potential-WHO Policy Perspectives on Medicines. $2002 ;$ No. 002.

4. Junsongduang A, Balslev $H$, Inta A, Jampeetong A, Wangpakapattanawong $P$ : Karen and Lawa medicinal plant use: Uniformity or ethnic divergence?. J. Ethnopharmacol. 2014; 151: 517-527.

5. Bye R A: Medicinal plants of the sierra madre: Comparative study of tarahumara and Mexican market plants. Econ. Bot 1986; 40: 103-124.

6. Yong G: The Hill Tribes in Northern Thailand. Siam Society. Bangkok, Thailand; 1962.

7. Johnson N: Environmental change in Northern Thailand, Impact on wild edible plant availability. Ecol. Food Nutr. 2002; 41: 373-399.

8. Junsongduang A, Balslev H, Inta A, Jampeetong A, Wangpakapattanawong P: Medicinal plants from swidden fallows and sacred forest of the Karen and the Lawa in Thailand. J. Ethnobiol. Ethnomed. 2013; 9: 44.

9. Khamfachuea K, Trisonthi P, Trisonthi C: Ethnobotany of the Karen at Ban Chan and Chaem Luang subdistricts, Mae Chaem district, Chiang Mai province. Thai J Bot 2010(Special Issue):275-297.

10. Tangjitman K, Wongsawad C, Kamwong K, Sukkho T, Trisonthi C: Ethnomedicinal plants used for digestive system disorders by the Karen of northern Thailand. J Ethnobiol Ethnomed 2015; 11(1):27.

11. Tangjitman K, Wongsawad C, Winijchaiyanan P, Sukkho T, Kamwong K, Pongamornkul W, Trisonthi C: Traditional knowledge on medicinal plant of the Karen in northern Thailand, A comparative study. J Ethnopharmacol 2013; 150(1):232-243.

12. Trisonthi C, Trisonthi P: Ethnobotanical study in Thailand, a case study in Khun Yuam District Maehongson Province. Thai J Bot 2009; 1(1):1-23.

13. The Plant List, 2013. Version 1.1, Available online: [http://www.theplantlist.org] (accessed on January 20, 2020)

14. Cook FE: Economic Botany Data Collection Standard: Royal Botanic Gardens (Kew); 1995.

15. Pooma R, Suddee S: Thai Plant Names Tem Smitinand. Forest Herbarium and Department of National Parks, Wildlife and Plant Conservation, Bangkok; 2014. 
16. Phillips O, Gentry AH, Reynel C, Wilkin P, Galvez-Durand BC: Quantitative ethnobotany and Amazonian conservation. Conserv Biol. 1994; 8 (1): 225-248.

17. Heinrich M, Ankli A, Frei B, Weimann C, Sticher O: Medicinal plants in Mexico: healers' consensus and cultural importance. Soc. Sci. Med. 1998; 47: 1859-1871.

18. Trotter RT, Logan MH: Informant consensus: a new approach for identifying potentially effective medicinal plants, in: N. L. Etkin (Ed.), Plants in Indigenous Medicine and Diet; Redgrave Publishing Company, Bedford Hills, New York; 1986.

19. Phumthum M, Srithi K, Inta A, Junsongduang A, Tangjitman K, Pongamornkul W, Trisonthi C, Balslev H: Ethnomedicinal plant diversity in Thailand. J Ethnopharmacol 2018; 214:90-98.

20. Inta A, Shengji P, Balslev, H, Wangpakapattanawong, P, Trisonthi C: A comparative study on medicinal plants used in Akha's traditional medicine in China and Thailand, cultural coherence or ecological divergence?. J. Ethnopharmacol. 2008; 116: 508-517.

21. Khuankaew S, Srithi K, Tiansawat P, Jampeetong A, Inta A, Wangpakapattanawong P: Ethnobotanical study of medicinal plants used by Tai Yai in northern Thailand. J Ethnopharmacol 2014; 151(2):829-838.

22. Inta A, Trisonthi P, Trisonthi C: Analysis of traditional knowledge in medicinal plants used by Yuan in Thailand. J Ethnopharmacol 2013; 149(1):344-351.

23. Shaheen H, Qaseem, MF, Amjad M S, Bruschi P: Exploration of ethno-medicinal knowledge among rural communities of Pearl Valley. Rawalakot, District Poonch Azad Jammu and Kashmir. PLoS One; $2017 ; 12$.

24. Ong HG, Kim Y-D : Quantitative ethnobotanical study of the medicinal plants used by the Ati Negrito indigenous group in Guimaras island, Philippines. J. Ethnopharmacol. 2014; 157: 228-242.

25. Tetali P, Waghchaure C, Daswani PG, Antia NH, Birdi TJ: Ethnobotanical survey of antidiarrhoeal plants of Parinche valley, Pune district, Maharashtra, India. J. Ethnopharmacol. 2009; 123: 229-236.

26. Mosaddegh M, Esmaeili S, Hassanpour A, Malekmohammadi M, Naghibi F: Ethnobotanical study in the highland of Alvand and Tuyserkan, Iran. Res. J. Pharmacogn. 2016; 3: 7-17.

27. Inta A, Sirisa-ard P, Pongamornkul W: Medicinal plants in Ban Hua Thung Community Forest, Chiang Dao Wildlife Sanctuary, Chang Dao District, Chiang Mai Province. Thai J. Bot 2012; 4: 213-232.

28. Noctor G, Foyer CH : ASCORBATE AND GLUTATHIONE: Keeping Active Oxygen Under Control. Annu. Rev. Plant Biol. 1998; 49: 249-279.

29. Azwanida NA: Review on the Extraction Methods Use in Medicinal Plants, Principle, Strength and Limitation. Med. Aromat. Plants 2015; 4: 2167-0412.

30. Anderson EF: Ethnobotany of hill tribes of northern Thailand 1, Medicinal plants of Akha. Econ. Bot. 1986a; 40: 38-53.

31. Anderson EF: Ethnobotany of hill tribes of northern Thailand 2, Lahu medicinal plants. Econ. Bot. 1986b; 40: 442-450.

32. Coe FG, Anderson GJ: Ethnobotany of the garífuna of Eastern Nicaragua. Econ. Bot. 1996; 50: 71-107.

33. Simbo DJ: An ethnobotanical survey of medicinal plants in Babungo, Northwest Region, Cameroon. J. Ethnobiol. Ethnomed. 2010; 6.

34. Kantasrila R, Pongamornkul W, Panyadee P, Inta A: Ethnobotany of medicinal plants used by Karen, Tak Province in Thailand. Thai J. Bot. 2017; 9 (2): 193-216.

35. Ribeiro JPdO, Carvalho TKN, Ribeiro JEdS, de Sousa RF, Lima JRdF, de Oliveira RS, Alves CAB, Jardim JG, de Lucena RFP: Can ecological apparency explain the use of plant species in the semi-arid depression of 
Northeastern Brazil?. Acta Bot. Bras. 2014; 28: 476-483.

36. Camou-Guerrero A, Reyes-García V, Martínez-Ramos M, Casas A: Knowledge and Use Value of Plant Species in a Rarámuri Community: A Gender Perspective for Conservation. Hum. Ecol. 2008; 36: 259-272.

37. Kayani S, Ahmad M, Zafar M, Sultana S, Khan MPZ, Ashraf MA, Hussain J, Yaseen G: Ethnobotanical uses of medicinal plants for respiratory disorders among the inhabitants of Gallies - Abbottabad, Northern Pakistan. J. Ethnopharmacol. 2014; 156: 47-60.

38. Sbai-Jouilil H, Fadli A, Hafian ME, Ayad RE, Benharbit O, Zidane L: Floristic and Ethnobotanical Study of Medicinal Plants Used in the Treatment of Respiratory Diseases in Seksaoua Region (Western High Moroccan Atlas). Annu. Res. Rev. Biol. 2017; 17: 1-10.

39. de Albuquerque UP: Re-examining hypotheses concerning the use and knowledge of medicinal plants: a study in the Caatinga vegetation of NE Brazil. J. Ethnobiol. Ethnomed. 2006; 2: 30-30.

\section{Figures}

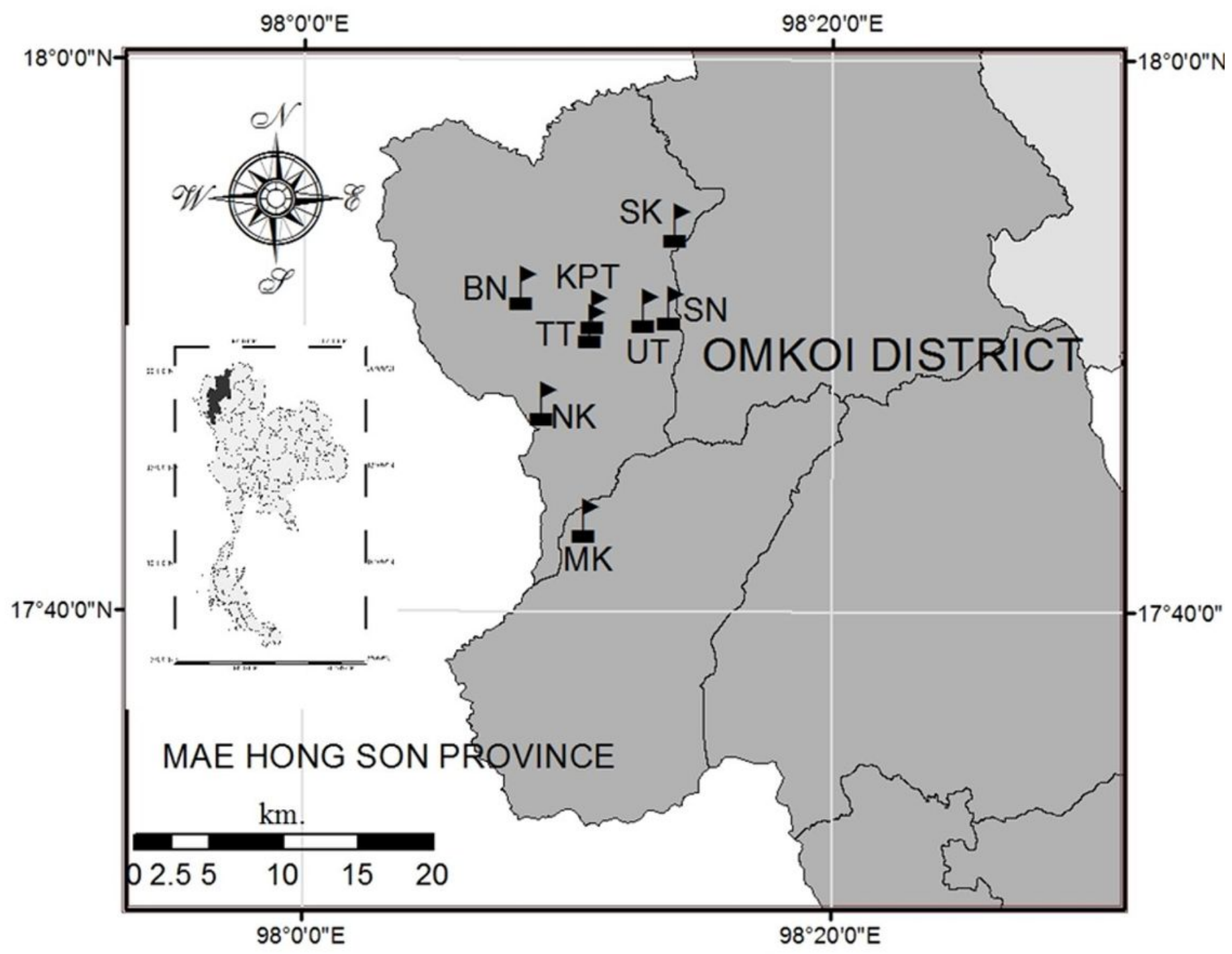

Figure 1 
Location of 8 Pwo villages in Na Kian sub-district, Omkoi district, Chiang Mai Province, Thailand where the medicinal plants were studied

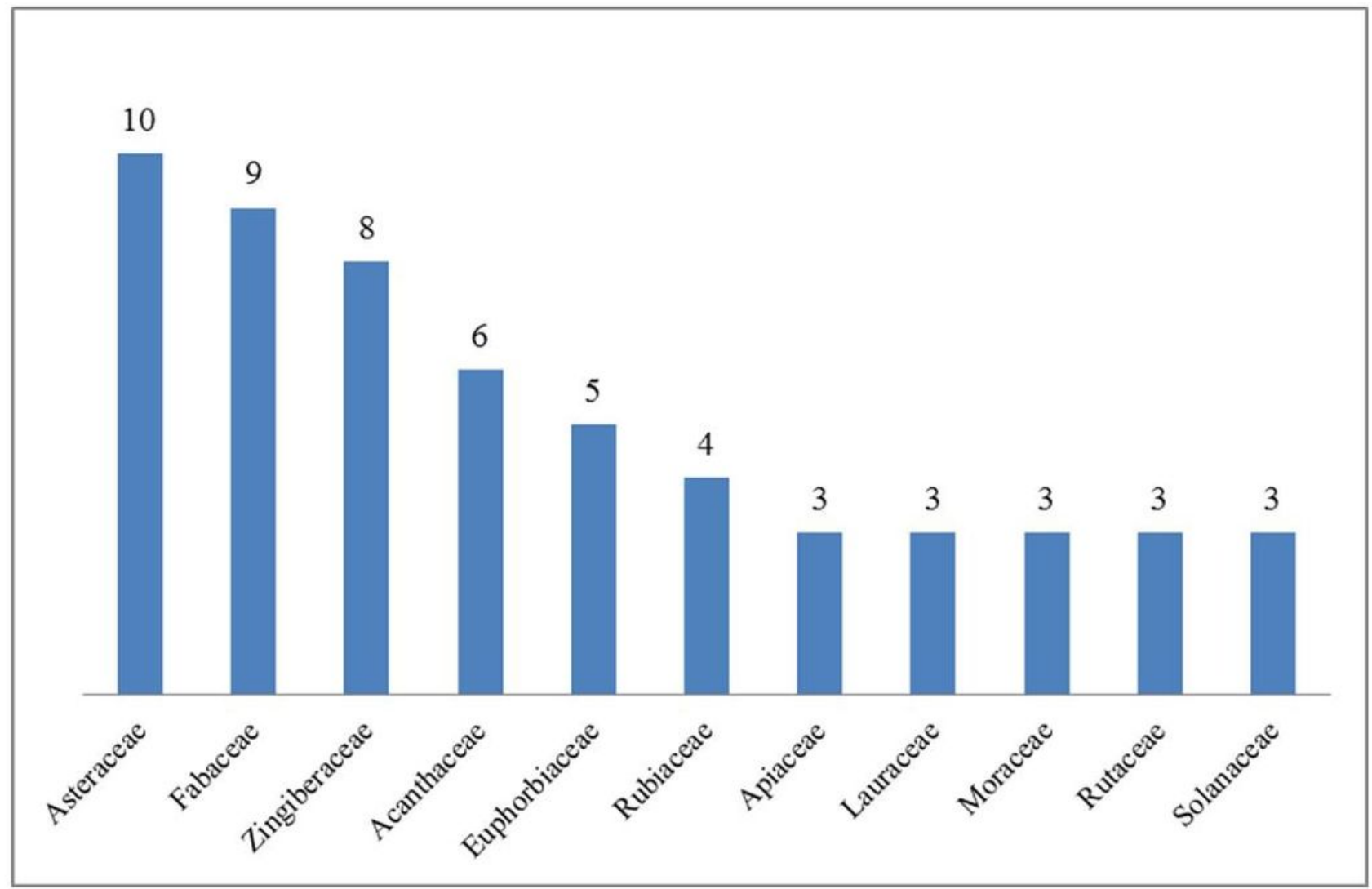

Figure 3

The number of ethnomedicinal plant species found in each family*. 


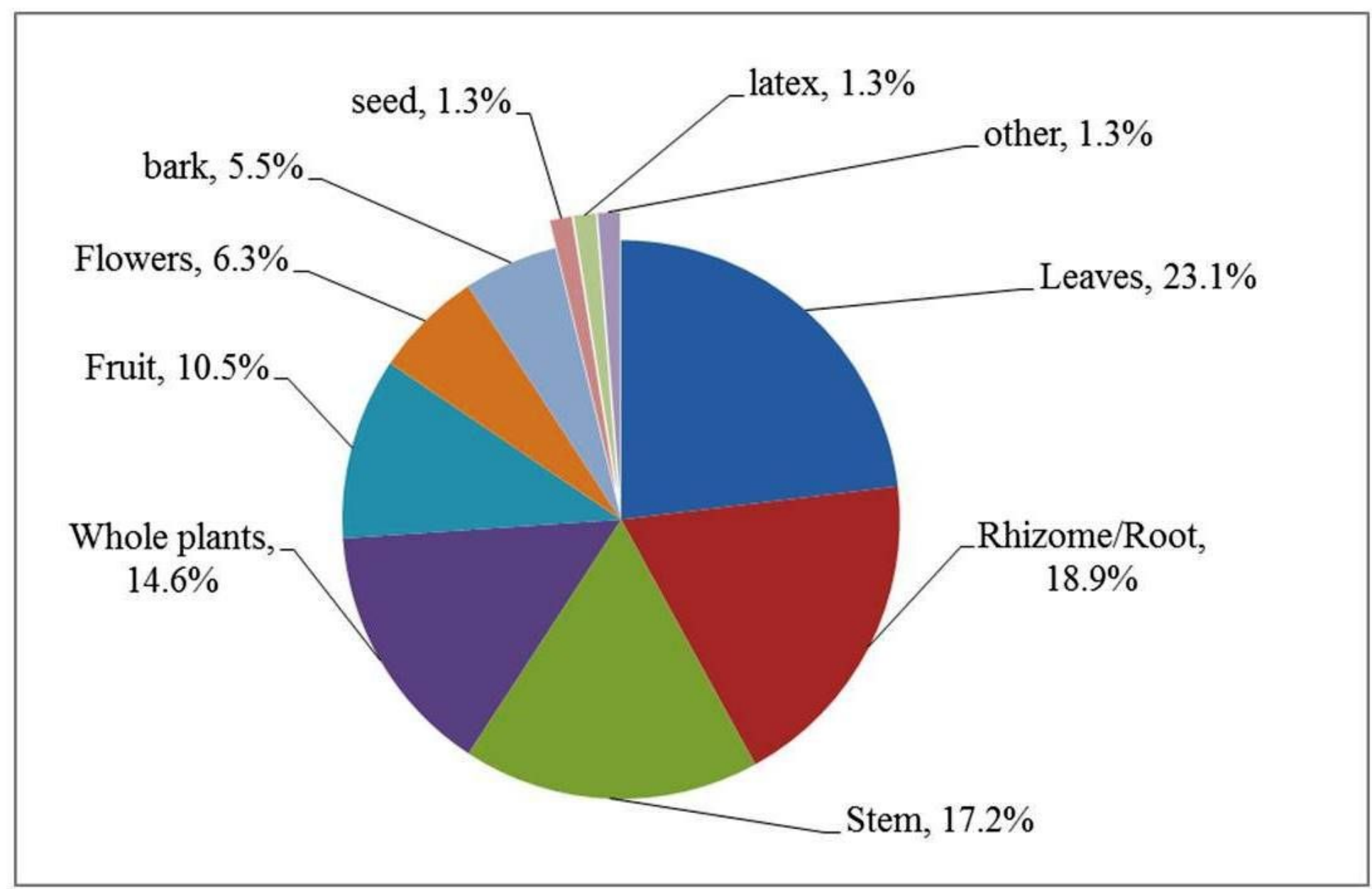

\section{Figure 5}

The percentage of each plant parts which were used by 50 Pwo informants, from 8 villages in Na Kian sub-district, Omkoi district, Chiang Mai provinces, Thailand. 


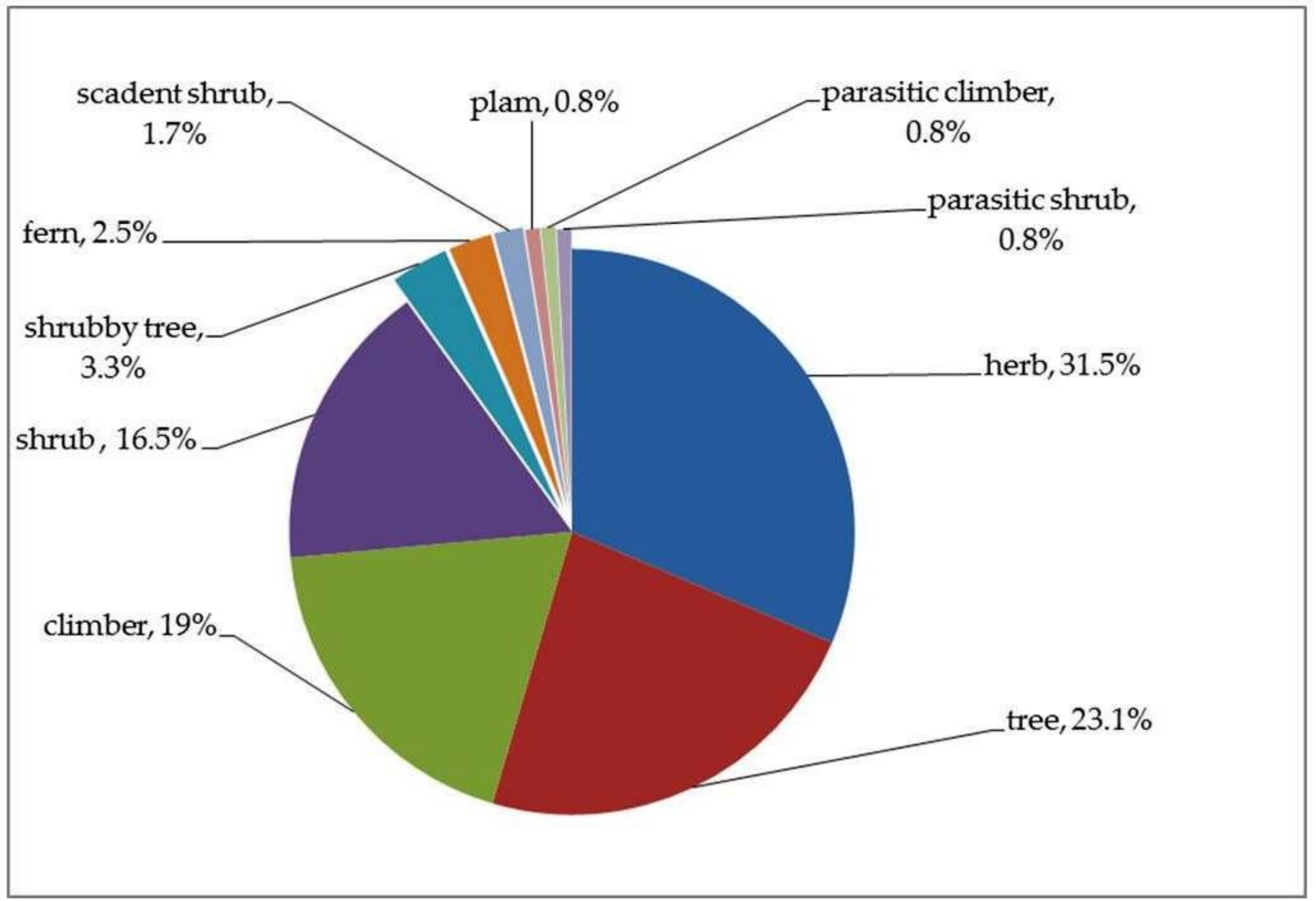

\section{Figure 7}

The percentage of each plant life from which were used by 50 Pwo informants, from 8 villages in Na Kian subdistrict, Omkoi district, Chiang Mai provinces, Thailand. 


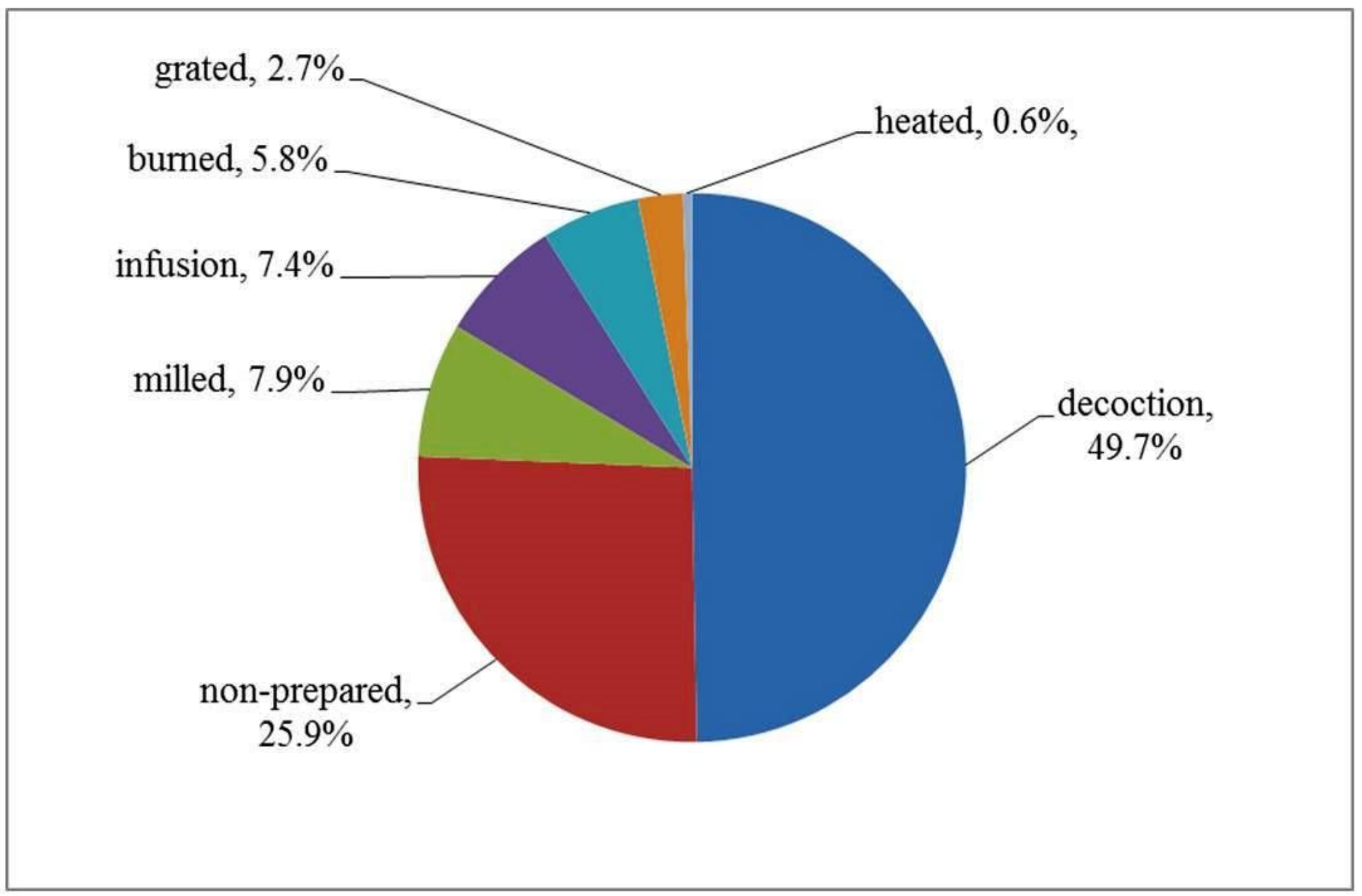

\section{Figure 9}

The percentage of each method of preparation which were used by 50 Pwo informants, from 8 villages in Na Kian sub-district, Omkoi district, Chiang Mai provinces, Thailand. 